\title{
MedienPädagogik
}

Zeitschrift für Theorie und Praxis der Medienbildung

\section{Editorial: Social Media in der Offenen Kinder- und Jugendarbeit}

Daniela Cornelia Stix

\begin{abstract}
Zusammenfassung
Das soziale und kommunikative Handeln zwischen den Menschen hat sich durch die Nutzung von Medien im Allgemeinen und Social-Media-Plattformen (kurz: SMP) im Besonderen in den vergangenen Jahren verändert. Auch das pädagogische Handeln von Fachkräften ist von diesen gesellschaftlichen Prozessen nicht ausgenommen. Die Fachkräfte, die Teams und die Träger müssen Strategien finden, mit den veränderten Rahmenbedingungen umzugehen.
\end{abstract}

SMP sind ein bedeutender Teil insbesondere im Leben von jungen Menschen und wenn pädagogische Fachkräfte ihrer Aufgabe, junge Menschen auf dem Weg zu einer gesellschaftsfähigen Persönlichkeit zu begleiten, nachkommen wollen, müssen sie proaktiv auf diese veränderten Bedingungen reagieren und SMP in ihr berufliches Handeln integrieren. Im Fokus dieser Arbeit steht daher die Nutzung von SMP als Instrumente pädagogischen Handelns in der Offenen Kinder- und Jugendarbeit (kurz: OKJA). Dieses anfänglich sehr offen formulierte Forschungsinteresse war dabei Symptom einer - bis heute noch immer - schwach ausgeprägten Forschungslandschaft hinsichtlich der Nutzung von SMP in der OKJA, die weit hinter die Nutzung(-sumfänge und -erfahrungen) in der Praxis zurückfällt. Eine Tatsache, die als solche bereits einen dringenden Forschungsbedarf begründet. Es fehl(t)en ein differenzierter Überblick und eine systematische Deskription der neuen, medialen Handlungspraxen von pädagogischen Fachkräften und ihrer Bedingungen. Folglich fehl(t)e auch eine auf Empirie basierende theoretisch fundierte Grundlage, die wiederum in der Praxis dazu hätte dienen können, das fachliche Handeln theoretisch rückzubinden. Die vorliegende Arbeit ist ein kumulatives, publikationsbasiertes Dissertationsprojekt. Angelehnt an den Forschungsstil der Grounded Theory wurden die verschiedenen Forschungsfragen sukzessive, aufeinander aufbauend aus zunächst fremden, später aus den eigenen empirischen Erkenntnissen und unter iterativer Rückbindung auf Theorien entwickelt. Das offen formulierte Forschungsinteresse, SMP als Instrumente pädagogischen Handelns in der OKJA untersuchen zu wollen, konkretisierte sich im Verlauf des Forschungsprozesses zunehmend hinsichtlich einer Professionalisierungsperspektive. Im Fokus stand unter anderem die Wahrnehmung des professionellen pädagogische Handelns durch die pädagogischen Fachkräfte, die Bedeutung für pädagogisches Handeln, SMP als Handlungsräume zu definieren, sowie die Entwicklung von Strategien, um professionellen Standards der Sozialen Arbeit unter den medialen Bedingungen gerecht zu werden.

In Kapitel 1 Entwicklung der Foki und Ziele werden die Entwicklungen der Forschungsfoki, -fragen und -ziele nachgezeichnet sowie die theoretischen und empirischen Zusammen-

Stix, Daniela Cornelia. 2020. «Editorial: Social Media in der Offenen Kinder- und Jugendarbeit». MedienPädagogik, (Social Media in der OKJA), 1-48. https://doi.org/10.21240/mpaed/diss.ds/2020.10.01.X. 
hänge zwischen den publizierten Beiträgen hergestellt. Die eigenen empirischen Daten entstammen episodischen Interviews, die mit pädagogischen Fachkräften aus Berliner Jugendfreizeiteinrichtungen (kurz: JFE) in den Jahren 2012/13 und 2017 geführt wurden. Die Datenauswertung erfolgte mittels offener und axialer Kodierung und anhand des Kodierparadigmas entsprechend der (Reflexiven) Grounded Theory. Um der Leserin, dem Leser der vorliegenden Forschungsarbeit zu ermöglichen, die im Forschungsprozess getroffenen Entscheidungen und Analysen nachzuvollziehen, wird die forschungsmethodische Umsetzung in Kapitel 2 Empirische Untersuchung detailliert begründet und dargelegt. Eine Zusammenfassung der Forschungsergebnisse aus den Teilpublikationen findet sich in Kapitel 3. Die Einzelergebnisse werden dort drei zentralen Themenbereichen zugeordnet. Aus diesen werden im anschliessenden 4. Kapitel Konsequenzen für das professionelle Handeln von pädagogischen Fachkräften abgeleitet sowie im 5. Kapitel Forschungsdesiderate formuliert. Das vorliegende Editorial endet mit einer tabellarischen Übersicht über die im Rahmen des kumulativen Dissertationsprojekts angefertigten und veröffentlichten Publikationen (Kapitel 6).

\title{
Social Media in Youth Work
}

\begin{abstract}
Social and communicative action between people has changed in recent years through the use of media in general and social media platforms (SMP for short) in particular. The pedagogical activities of professionals are also not excluded from these social processes. Professionals, teams and social work organizations have to find strategies to deal with the changed framework conditions.

SMP are an important part of young peopless lives in particular and if educational professionals want to fulfil their task of accompanying young people on their way to becoming socially acceptable personalities, they must react proactively to these changed conditions and integrate SMP into their professional activities.

The focus of this work is therefore the use of SMP as instruments of pedagogical action in youth work (Offene Kinder und Jugendarbeit in short: OKJA). This initially very openly formulated research interest was a symptom of - still today - a weak research landscape with regard to the use of SMP in OKJA, which lags far behind the use (scope and experience) falls behind in practice. A fact which as such already establishes an urgent need for research. A differentiated overview and a systematic description of the new, media-based practices of youth workers and their conditions is missing. Consequently, a theoretically sound empirical foundation is also lacking, which in turn could have served in practice to theoretically re-integrate professional action.
\end{abstract}


The present work is a cumulative, publication-based dissertation project. In keeping with the research style of Grounded Theory, the various research questions were developed successively, building on each other from initially literature research, and later from the authors own empirical findings and with iterative reference to theories. The openly formulated research interest in investigating SMP as instruments of pedagogical action in the OKJA became increasingly concrete in the course of the research process with regard to a professionalisation perspective. The focus was, among other things, on the perception of professional pedagogical action by the pedagogical staff, the importance for pedagogical action of defining SMP as areas of action, as well as the development of strategies to meet professional standards of social work under media conditions.

In Chapter 1 Entwicklung der Foki und Ziele, the developments of the research focus, questions and objectives are traced and the theoretical and empirical connections between the published contributions are established. My empirical data are taken from episodic interviews conducted with educational specialists from Berlin youth leisure facilities (in short: JFE) in 2012/13 and 2017. The data evaluation was carried out by means of open and axial coding and on the basis of the coding paradigm according to the (Reflective) Grounded Theory. In order to enable the reader to understand the decisions and analyses made in the research process, the methodological implementation of the research is explained and explained in detail in Chapter 2 Empirische Untersuchung. A summary of the research results from the sub-publications can be found in Chapter 3, where the individual results are assigned to three central topic areas. From these, consequences for the professional actions of educational specialists are derived in the subsequent 4th chapter and research desiderata are formulated in the 5th chapter. The present editorial ends with a tabular overview of the publications produced and published in the context of this cumulative dissertation project (Chapter 6).

\section{Lesehinweise}

Um die Doppelnennung Kinder und Jugendliche, als Bezeichnung für die Besucherinnen und Besucher von Einrichtungen der Offenen Kinder- und Jugendarbeit, zu vermeiden, werden diese als junge Menschen bezeichnet.

Im Rahmen des vorliegenden Forschungsprojekts wurde zu Beginn die Bezeichnung Soziale Onlinenetzwerke (SONW) verwendet. Das zum damaligen Zeitpunkt populärste SONW war Facebook. Die steigende Nutzung von Messenger-, Bild und Videodiensten (wie WhatsApp, Instagram, YouTube) und die Abnahme der Facebooknutzung (vgl. Medienpädagogischer Forschungsverbund Südwest 2013) machten es notwendig, den Fokus auf Social-Media-Plattformen (SMP) zu öffnen, um die Aktualität der Ergebnisse der vorliegenden Arbeit zu gewährleisten. In den Publikationen, die diese Arbeit enthalten, wurde folglich in Beiträgen, die ab 2017 verfasst wurden, die Sammelbezeichnung SMP verwendet. 
Social-Media-Plattformen werden definiert als die digitalen Medien, die es Nutzenden ermöglichen, im Internet Inhalte zu gestalten, sie anderen zu präsentieren und sich (darüber) miteinander auszutauschen. SMP umfassen verschiedene Möglichkeiten der Interaktion wie beispielsweise SONW, Wikis, (Micro-)Blogs oder Social Sharing (vgl. Ebersbach et al. 2011). In der vorliegenden Arbeit werden auch Messengerdienste den SMP zugeordnet, da die Anwendungen technisch zunehmend verschmelzen (vgl. Ebersbach et al. 2011, 115).

Zugunsten der Lesbarkeit und des Textumfangs werden die folgenden Abkürzungen verwendet:

- OKJA: Offene Kinder- und Jugendarbeit

- JFE: Jugendfreizeiteinrichtungen

- SONW: Soziale Onlinenetzwerke (bis 2017)

- SMP: $\quad$ Social-Media-Plattformen (ab 2017)

\section{Entwicklung der Foki und Ziele}

Der Auslöser, sich mit der Thematik Social Media in der Offenen Kinder- und Jugendarbeit (kurz OKJA) zu beschäftigen, basiert auf meinen berufsbiografischen Erfahrungen. Als Medienpädagogin in einem Berliner Medienkompetenzzentrum pflegte ich ein enges Netzwerk zu den Einrichtungen der OKJA (im Folgenden bezeichnet als Jugendfreizeiteinrichtungen, kurz JFE) in meinen Bezirken und konnte beobachten, wie unterschiedlich die pädagogischen Fachkräfte auf den «Trend» Social-Media-Plattformen (kurz SMP) reagierten. Die Reaktionen reichten von Ablehnung und Desinteresse bis zu völliger Begeisterung und nahezu sofortiger, funktionalisierender Implementierung in den beruflichen Alltag.

In einer ersten empirisch fundierten Deskription der Nutzung von SMP in der ausserschulischen Jugendarbeit findet sich dieses Reaktionsspektrum wieder (Korfmacher 2011) $)^{1}$. Von den 140 Befragten gibt gut die Hälfte an, SMP bereits in der Arbeit zu nutzen. Informationsverbreitung und -erhalt, Dokumentationen sowie allgemeiner kommunikativer Austausch und Beratungsgespräche stehen dabei im Fokus der Nutzung (Korfmacher 2011, 12). Die durchschnittliche Zeit, die pro Tag für die Pflege und Kommunikation auf SMP aufgebracht wird, umfasst 2,1 Stunden, wobei die vorhandene Zeit jeweils hälftig als ausreichend und als ungenügend eingeschätzt wird (Korfmacher 2011, 13). Als grösste Hemmnisse für die Arbeit werden vor allem zu geringe personelle und zeitliche Ressourcen genannt. Auch rechtliche Unsicherheiten

1 Das Landesprogramm jugendnetz-berlin gab die Studie im Jahr 2011 in Auftrag. Ziel war es, einen Überblick zur Situation der «Online-Jugendarbeit» zu erhalten und mögliche Probleme und Unterstützungsbedarfe zu identifizieren sowie Felder mit Verbesserungsbedarf aufzuzeigen (Korfmacher 2011, 4f.). Die Daten wurden mittels Onlinefragebogen auf Grundlage der Software LimeSurvey erhoben. Dieser war für die Dauer von 6 Wochen im Zeitraum ab März 2011 online gestellt und per E-Mail zur Teilnahme aufgefordert. Mit einer Rücklaufquote von rund 20 Prozent standen 140 ausgefüllte Fragebogen zur Auswertung zur Verfügung (Korfmacher 2011, 6). 
und Vorbehalte im Team stellen laut Korfmacher ein Problem bei der Onlinearbeit dar (Korfmacher 2011, 18). Weitere 16 Prozent geben an, die Arbeit im Web sei «für die Zielgruppe nicht relevant» (Korfmacher 2011, 18).

Geht man technisch und zeitlich etwas weiter zurück, findet sich ein ähnlich ausgefächertes Spektrum hinsichtlich der Einschätzungen von Sozialarbeitenden zur Bedeutung des Internets für die Soziale Arbeit (Wittenberg et al. 2001)²: Die Fachkräfte sehen einerseits verschiedene Chancen für die Klientinnen und Klienten als auch für die Kommunikation mit Kolleginnen und Kollegen sowie Vorgesetzten. Als Risiken wird befürchtet, dass es datenschutzrechtliche Probleme geben wird, vermehrte Leistungserschleichungen stattfinden sowie der abnehmende persönliche Kontakt zu Qualitätsverlusten führt (Wittenberg et al. 2001, 50ff.). Ein grosser Teil der Einrichtungen verfügt bereits über ein eigenes Informationsangebot im Internet. Mit der Realisierung eines solchen Auftritts verbundene Probleme sind die Bewältigung der Informationsflut und die redaktionelle Pflege des Angebots. Die Akzeptanz des Angebots durch Kolleginnen und Kollegen wird problematischer als die Akzeptanz durch Klientinnen und Klienten bewertet. Die entstehenden Kosten stehen demgegenüber etwas weniger im Vordergrund. Die technische Realisierung eines solchen Angebotes wird weitgehend für unproblematisch gehalten (Wittenberg et al. 2001, 61ff.). Dagegen wird aber eine Eruption des bisherigen Grundverständnisses der Sozialen Arbeit befürchtet (Professionalitätsverlust, Unpersönlichkeit, Wertverlust, Ausgrenzung bestimmter Klientinnen und Klienten sowie Ökonomisierung Sozialer Arbeit) (Wittenberg et al. 2001, 66f.).

Die Forscher weisen in ihrem Fazit darauf hin, dass die befragten Fachkräfte zwar einen grossen Bedeutungszuwachs von Informations- und Kommunikationstechnologien für die Soziale Arbeit sehen und dies Veränderungen des pädagogischen Handelns mit sich bringe. Jedoch geben sie auch zu bedenken, dass die verstärkte Nutzung nicht allein aus ihrer Eigendynamik heraus sinnvoll sei. Je nach sozialpädagogischem Bereich müsse abgewogen und die optimale Nutzung zugeschnitten werden (Wittenberg et al. 2001, 68).

Der sich in den obigen Studien andeutende Wandel wird von Krotz als Mediatisierung bezeichnet (2001a). Krotz untersuchte, wie Alltag, Identität, Kultur und Gesellschaft durch die Entwicklung der Medien beeinflusst werden (vgl. Krotz 2006, 62). Er kommt zu der Erkenntnis, dass Medien auf zwei Wegen wirken. Zu einem geringen Teil wirken sie über die präsentierten Inhalte, zu einem grösseren Teil durch ihre

2 Wittenberg et al. waren unter den ersten, die in Deutschland die Nutzung von Informations- und Kommunikationstechnologien (IKT) in Feldern der Sozialen Arbeit untersuchten. Ziel ihrer Umfrage war eine systematische Aufarbeitung der bisherigen Erfahrungen der (pädagogischen) Fachkräfte in Bezug auf die Nutzung des Internets im Bereich der Sozialen Arbeit. Des Weiteren sollten sich daraus ergebende Möglichkeiten, Grenzen, Folgen und Prognosen empirisch basiert eingeschätzt werden können (Wittenberg et al. 2001, 42). Dazu wurde im Herbst 1999 eine Onlineumfrage durchgeführt, zu der mittels Mailinglisten eingeladen wurde (Wittenberg et al. 2001, 43). Als Datengrundlage konnten 156 Fragebogen analysiert werden (Wittenberg et al. 2001, 44). 
«Kommunikationspotenziale». Dies bedeutet, sie wirken «durch die Tatsache, dass die Menschen sich selbst durch deren Verwendung in spezifischer Weise zurichten, ihr Beziehungsnetz und ihren realisierten Handlungsraum daran orientieren und sich selbst und ihre Gesellschaft ebenso wie Identität und Kultur als Sinnsystem auf veränderte Weise produzieren» (vgl. Krotz 2007, 12 sowie Krotz 2006, 62). Mit anderen Worten sind die medientechnischen Entwicklungen und ihre kulturellen und gesellschaftlichen Konsequenzen für Krotz vor allem ein soziales Geschehen. Ein Wandel ergibt sich dadurch, dass immer mehr Menschen ihre Mediennutzungsgewohnheiten und Interessen ausdifferenzieren. Zunehmend beziehen sie ihr soziales und kommunikatives Handeln auf eine grössere Anzahl von Medien (Krotz 2001).

Die Mediatisierung führt laut Krotz dabei besonders zu jugendkulturellen Veränderungen. Er stellt fest, dass sich derzeit ein Wandel abzeichnet, der darauf verweist, "dass sich Jugendkulturen heute sehr viel grundlegender mit und über Medien konstituieren als in der Vergangenheit» (Krotz und Schulz 2013, 34). Den technischen Ausgangspunkt sieht er in der Digitalisierung und Konvergenz von Medien sowie der damit einhergehenden Entstehung neuer «Kommunikationspotenziale» (Krotz 2001). Jugendliche eignen sich Medien an, machen sie sich für ihre Zwecke zunutze und entwickeln somit eine selbstverständliche Alltagspraxis.

Krotz' Analyse zur Bedeutung von Medien wird durch die aktuelle JIM-Studie des Medienpädagogischen Forschungsverbund Südwest ${ }^{3}$ unterstrichen, der zufolge die befragten jungen Menschen angaben, im Schnitt täglich dreieinhalb Stunden online zu sein (MPFS 2018, 31). Die liebsten Internetangebote und Apps sind derzeit YouTube, WhatsApp und Instagram (MPFS 2018, 32, offene Abfrage).

Für pädagogische Fachkräfte der OKJA, die mit den jungen Menschen arbeiten, führt der Prozess der Mediatisierung zu einer Veränderung der pädagogischen Rahmenbedingungen, der Anlässe, auf die sie reagieren, sowie der Formen, mit denen sie den Gegenstand bearbeiten. Das soziale und kommunikative Handeln zwischen den Menschen hat sich durch die Nutzung von Medien im Allgemeinen und SMP im Besonderen in den vergangenen Jahren verändert. Kommunikation findet global, frei von zeitlichen und räumlichen Beschränkungen statt und beeinflusst zunehmend die zwischenmenschlichen Interaktionen. Die pädagogischen Fachkräfte müssen auf diese veränderten Bedingungen reagieren, wenn sie junge Menschen auf dem Weg zu einer gesellschaftsfähigen Persönlichkeit begleiten wollen.

Wie die obigen Untersuchungen gezeigt haben, ist die OKJA bereits auf vielfältige Art von Medien durchdrungen und die Mediatisierung hat möglicherweise tiefgreifende Veränderungen für die Handlungsfelder der Sozialen Arbeit eingeläutet. Kutscher et al. veranschaulichen diesen Prozess anhand ihres Modells der «Mediatisierungsdimensionen in der Sozialen Arbeit». Beispielsweise tauschen sich Professionelle

3 Auch die jährlichen Untersuchungsergebnisse in der ARD-ZDF-Onlinestudie (http://www.ard-zdf-onlinestudie.de) sowie des Digital Index (ehemals (N)Onliner Atlas, https://initiatived21.de/studien) bestätigen die folgenden Zahlen. 
untereinander in Fachforen oder über Mailinglisten aus, bieten den Klientinnen und Klienten Onlineberatung sowie Partizipationsmöglichkeiten und stellen ihnen relevante Informationen zur Verfügung (Kutscher et al. 2015, 4). Auch der Rückgriff auf Fachsoftware bei Prozessen der Diagnostik, Planung, Dokumentation und Evaluation von Hilfen zeugt von der Mediatisierung der Sozialen Arbeit (Kutscher et al. 2015, 3f.).

$\mathrm{Zu}$ diesen Entwicklungen steht jedoch empirische Forschung noch weitgehend aus. Kutscher et al. kritisieren, dass es bislang keinen systematischen Überblick zu den Folgen der Mediatisierung hinsichtlich der Lebenswelten der Klientinnen und Klienten, der Arbeitswelt und der Handlungspraxen der Fachkräfte gibt (Kutscher et al. 2015, 7). Insbesondere im Hinblick auf die Einflüsse der Mediatisierung auf die Handlungspraxen von pädagogischen Fachkräften liegt kaum empirisches Material vor.

Die genannten Aspekte fokussieren auf ein Phänomen, das sich für Empirie, Theorie und Praxis somit als relevant darstellt. Für die vorliegende Forschungsarbeit ergibt sich daraus als Ausgangspunkt das folgende Forschungsinteresse:

SMP als Instrumente pädagogischen Handelns in der OKJA.

Dieses Interesse als Ausgangspunkt nehmend stellte sich die Frage nach der spezifischen Datenlage im Handlungsfeld der OKJA und die Aufgabe deren systematischer Aufbereitung.

\subsection{Datenlage und Forschungsdesiderate}

Eine systematische Erfassung der Datenlage (siehe «Warum Soziale Onlinenetzwerke in der Jugendarbeit noch weiter erforscht werden müssen») unterstrich die Notwendigkeit, dieses Forschungsinteresse zu verfolgen. Es zeigte sich, dass die Landkarte zum Thema SMP und pädagogisches Handeln im Kontext OKJA noch viele weisse Flecken aufweist. Augenfällig war, dass einer grossen Anzahl von Erfahrungsberichten eine je extrem geringe Anzahl von theoretischen und empirischen Arbeiten gegenüberstehen.

Letztere konnten aufzeigen, dass sich sozialräumliche Konzepte potenziell auf die (pädagogische) Arbeit mit SMP übertragen lassen. Jedoch erfordert dies eine intensive Auseinandersetzung mit eigenen (pädagogischen) Motiven und Intentionen. Ein Profil anzulegen und die Funktionen von SMP zu nutzen, genügt dem nicht. Entscheidend im Sinne eines performativen Raumverständnisses ist die Interaktion, also das Interagieren der pädagogischen Fachkräfte mit den jungen Menschen in/ auf einer SMP. Als Forschungsdesiderate identifizierte Themen waren folglich Fragen nach der Professionalisierung pädagogischen Handelns, aber ebenso für die Praxis relevante Fragen nach Grenzen des Handelns sowie einem neu zu definierenden Nähe-Distanz-Verhältnis. 
Auch Alfert bemängelt in ihrer 2015 erschienenen Untersuchung ${ }^{4}$, dass ein blosses Dabeisein nicht mehr genüge und das Handeln der pädagogischen Fachkräfte im Kontext SMP einer Professionalisierung bedarf. Alfert fand beispielsweise heraus, dass nahezu die Hälfte der pädagogischen Fachkräfte, die Facebook nutzt, ihr privates Profil für berufliche Zwecke verwendet (Alfert 2015, 259). Auch fehlten in 61,2 Prozent der Einrichtungen konzeptionelle Fundierungen der Facebook-Nutzung (Alfert 2015, 264f.). Wo es Zielformulierungen gab, beziehen sich diese nahezu alle auf administrative Tätigkeiten (Bekanntmachung von Angeboten und Öffentlichkeitsarbeit). Die Kontakt- und Kommunikationsmöglichkeiten (Medienkompetenzvermittlung, Stärkung der Arbeitsbeziehungen oder Fachaustausch) werden von den pädagogischen Fachkräften praktisch kaum und Partizipationsmöglichkeiten gar nicht genannt (Alfert 2015, 268). Als bedenkenswert bezeichnet Alfert zudem die Erkenntnis, dass von den für die Administration der Facebook-Präsenz Hauptverantwortlichen Dreiviertel den Unterschied zwischen Profil und Seite, und damit deren unterschiedliche Interaktionsfunktionen, nicht kennen (Alfert 2015, 264).

Einerseits stellt die Auseinandersetzung mit SMP im Allgemeinen und Facebook im Speziellen für die pädagogischen Fachkräfte ein bedeutsames Thema dar (Alfert 2015, 260) und nimmt einen grossen Stellenwert in den Einrichtungen ein (Alfert 2015, 261). Andererseits schliesst Alfert aufgrund des grossen Vorkommens von fehlenden Zielbestimmungen und Nicht- oder offenkundig falschem Wissen zu Facebookfunktionen ${ }^{5}$ auf eine weitgehend intuitive Nutzung durch die pädagogischen Fachkräfte (Alfert 2015, 265). Die moniert daher: «Grundsätzlich sollte [...] Facebook nicht nur nebenbei genutzt werden. Es geht nicht bloss darum, dort zu sein, wo sich die Adressat_innen aufhalten, sondern hinter der Nutzung sollte eine systematische Handlung stecken, die Ziele verfolgt und anhand derer im besten Fall Wirksamkeitsfaktoren abzulesen sind» (Alfert 2015, 265).

Wichtig sei auch zu reflektieren, dass eine berufliche Nutzung grundsätzlich andere Herangehensweisen und anderes Wissen verlange. Es könne nicht davon ausgegangen werden, dass eine private Nutzungskompetenz automatisch genügend berufliche Nutzungskompetenzen mit sich bringe (Alfert 2015, 281).

4 Alfert (2015) untersuchte in ihrer Dissertation, wie Facebook in der Kinder- und Jugendhilfe zum damaligen Zeitpunkt genutzt wurde und leitete Herausforderungen und Unterstützungsbedarfe der pädagogischen Fachkräfte ab. Ziel war es, einen theoretischen und empirischen Überblick über die berufliche Facebooknutzung zu liefern und konkrete Problemfelder aufzuzeigen. Dazu verteilte Alfert im Jahr 2012 auf einer Fachtagung mit dem Titel «Gute Freunde? Social-Media und Soziale Arbeit. Neue Wege der Kommunikation> mit der Tagungsmappe entsprechende Fragebögen. Bei Tagungseröffnung wurden die 140 teilnehmenden pädagogischen Fachkräfte gebeten, sich an der Befragung zu beteiligen sowie wiederholt über die Twitterwall dazu motiviert (Alfert 2015, 246ff.). Grundlage der Datenanalyse bildeten nach dem Setzen eines Filters, der die Kinder- und Jugendarbeit fokussierte, 68 ausgefüllte Fragebögen (Alfert 2015, 257ff.).

5 Beispielsweise geben 16 pädagogische Fachkräfte an, (mehrmals) täglich bis wöchentlich mit den jungen Menschen zu chatten, und weitere 11 monatlich oder seltener. Jedoch haben nur 15 Personen angegeben, Facebook über ein privates Profil zu nutzen, was bedeutet, dass nur diesen Personen die ChatFunktion zur Verfügung steht (vgl. Alfert 2015, 272). 
Ausgehend von der obigen Datenlage, die durch die später erschienene Studie von Alfert unterstrichen wurde, konnte das zentrale Forschungsinteresse der vorliegenden Arbeit thematisch auf den Professionalitätsaspekt zugeschnitten werden und lautete somit:

SMP und professionelles pädagogisches Handeln von pädagogischen Fachkräften im Kontext OKJA.

Damit war auch die Forschungsperspektive auf die Sicht der pädagogischen Fachkräfte festgelegt. Die Perspektive der jungen Menschen sollte dennoch nicht unberücksichtigt bleiben. Sie galt als eine Art Legitimationsprüfung des Forschungsinteresses hinsichtlich eines pädagogischen Handelns auf SMP.

\subsection{Pädagogisierung von Jugendräumen und Grenzüberschreitungen}

SMP leben von den Informationen und Inhalten, die die Menschen auf ihnen veröffentlichen. Je mehr (Inter-)Aktionen stattfinden, desto attraktiver und wirtschaftlich wertvoller werden die Plattformen. Das Motto von Facebook lautete sinnlogisch «Für eine offene und vernetzte Welt» (Coyle 2011). Die Voreinstellungen der meisten SMP sind dementsprechend so angelegt, dass in das persönliche Profil eingetragene Informationen öffentlich einsehbar sind.

Der JIM-Studie von 2013 zufolge hat der Grossteil der jungen Menschen diese auf Facebook hinterlegten Informationen nur für ihre Freunde sichtbar geschaltet (MPFS 2013, 42). Dies wird aber wieder ad absurdum geführt, wenn man bedenkt, dass eine Freundesliste durchschnittlich 290 Personen umfasst (MPFS 2013, 39). Die daraus folgende Konsequenz haben Karl/Peluchette treffend zusammengefasst:

"As a result, friends now means a hodgepodge of real friends, former friends, friends of friends, coworkers or colleagues, relatives of all ages, and perhaps even a boss or professor. Thus, for most Facebook members (80\%), there is no separation between the backstage and the front stage» (Karl und Peluchette 2011, 215).

Karl und Peluchette schildern ein «Mischmasch» aus Freunden, ehemaligen Schulkameraden, Familienmitgliedern und Arbeitskollegen oder Vorgesetzten. Bezogen auf die vorliegende Arbeit könnte man diese Liste noch ohne weiteres auch um Lehrpersonen oder pädagogische Fachkräfte der ausserschulischen Bildungsarbeit wie der OKJA erweitern. Die wenigsten nutzen die von Facebook offerierten Funktionen und nehmen eine Hierarchisierung der einzelnen Personen des Netzwerks vor. Dies führt nicht nur zu Vermischungen von privaten und beruflichen Aspekten, sondern kann auch zu unbeabsichtigten oder unbewussten Grenzüberschreitungen führen. 
Goffman hat im Zusammenhang mit dem Thema des professionellen und privaten Rollenhandelns den Begriff der «critical barrier» etabliert. Sie trennt die sogenannte «front-stage» von der «back-stage» (dt. «Vorder- und Hinterbühne») (Goffman 1959). Handeln auf der Frontstage (also beispielsweise in öffentlichen oder professionellen Kontexten) unterscheidet sich von privatem Handeln und Menschen lassen nur wenige Personen in ihrem Leben in den sogenannten Backstagebereich. Bei Grenzüberschreitungen kommt es zu einer «spoiled performance», also einer Schädigung der Frontstage-Rolle oder des Frontstage-Auftretens (Goffman 1959).

Nutzen pädagogische Fachkräfte SMP im Rahmen ihrer Arbeit, erhalten sie andere Einblicke in den Backstagebereich von jungen Menschen, als diese ihnen verbal mitteilen. Um zu prüfen, ob pädagogisches Handeln auf SMP durch die jungen Menschen gebilligt wird - und das Forschungsinteresse somit in gewissem Masse eine legitime Grundlage hat -, wurde in der vorliegenden Arbeit also zunächst die folgende Frage gestellt:

Inwiefern ist es angemessen, als pädagogische Fachkraft der OKJA auf SMP zu agieren?

In Form einer umfassenden Recherche wurden zur Beantwortung dieser Frage empirische Studien zusammengetragen und systematisiert. In der Publikation «Soziale Onlinenetzwerke - (k)eine pädagogenfreie Zone?» werden diese dargelegt und zusammenfassend festgestellt, dass SMP von jungen Menschen nicht (mehr) als reine Jugendräume wahrgenommen werden. Argumente, die die jungen Menschen gegen eine (pädagogische) Nutzung von SMP durch Lehrpersonen vorbringen, haben übertragen auf die pädagogischen Fachkräfte der OKJA kein Gewicht. Denn die OKJA stellt mit ihren Strukturmerkmalen Lebensweltorientierung, Partizipation, Freiwilligkeit, Offenheit und Niedrigschwelligkeit sowie Machtarmut und Diskursivität einen besonderen Rahmen für eine pädagogische Nutzung von SMP dar.

Dies - so die Kernerkenntnis - spricht für eine Nutzung durch die pädagogischen Fachkräfte der OKJA. Eine weitere Erkenntnis ist, dass das Strukturmerkmal der Diskursivität bedeutsam ist, denn gemeinsam ausgehandelte Regeln zum Umgang mit SMP sind die Basis für ein akzeptierendes Miteinander. Die Billigung des pädagogischen Handelns auf SMP durch junge Menschen legitimiert zugleich das zuvor entwickelte Forschungsinteresse.

\subsection{Funktionen von Social-Media-Plattformen in der OKJA}

Wie die angeführten Studien von Wittenberg et al., Korfmacher und Alfert zeigen, schreiben die pädagogischen Fachkräfte den Medien bestimmte Funktionen in alltäglichem Ablauf und Tätigkeiten zu. Das Internet wird gesehen als Informationsquelle und Informationsdistributionsplattform, als Werkzeug für kollegiale 
Zusammenarbeit, als Werkzeug für administrative und kommunikativ-sozialarbeiterische Tätigkeiten in Verbindung mit zeitlichen und finanziellen Einsparmöglichkeiten (Wittenberg et al. 2001). SMP werden ähnlich genutzt: Sie dienen überwiegend zur Informationsverbreitung oder zum Erhalt von Informationen und werden darüber hinaus für alltägliche oder Beratungsgespräche sowie zur Dokumentation der Arbeit genutzt (Korfmacher 2011, 12). Laut Alfert werden die Bekanntmachung von Angeboten und die Öffentlichkeitsarbeit in Einrichtungskonzepten mittels SMP besonders häufig genannt (Alfert 2015, 268). Sowohl das Internet als auch SMP werden von den pädagogischen Fachkräften also für bestimmte (pädagogische) Zwecke funktionalisiert.

Ein Modell zur Funktionalisierung von Medien hat McQuail in seinem 1983 entwickelten Advocate-Ansatz entwickelt (McQuail 1983, 73ff.). Er unterscheidet insgesamt vier Positionen, die Medien für ihre Zwecke funktionalisieren: die Gesellschaft, Medienproduzenten, die Rezipienten sowie die sogenannten Advocates. Letztere definiert er als «representative of an institution or organized group which seeks benefit from using the media to reach public» (McQuail 1983, 75). In diesem Sinne kann man pädagogische Fachkräfte, die SMP ebenfalls zielgerichtet im Interesse ihrer jeweiligen Einrichtungen nutzen, als Advokaten bezeichnen. Nach McQuail erfüllen Medien für Advokaten die folgenden Funktionen:

Information: über die Einrichtung und ihre Aktivitäten sowie über relevante externe Geschehnisse informieren, Bildungs- und Informationskampagnen

Interpretation: relevante Informationen filtern, Öffentlichkeitsarbeit, Reputationsaufbau

Expression: Position und Grundsätze veröffentlichen, die Entwicklung eines Zugehörigkeitsgefühls fördern

Mobilisierung: zu Beteiligung und Unterstützung anregen, die Selbstorganisation der Anhänger unterstützen (McQuail 1983, 80f., auszugsweise übersetzt DCS)

Es fällt auf, dass sich einige dieser Funktionsbeschreibungen als pädagogische oder pädagogisch relevante Tätigkeiten bezeichnen lassen. In der Fachliteratur wird in der Regel der Begriff pädagogisches Handeln verwendet. Laut Giesecke habe sich die Perspektive pädagogischen Handelns zu einer partikularen verschoben, da jede/r für seine eigene Entwicklung verantwortlich sei. Die zentrale Aufgabe pädagogischer Fachkräfte sei somit die positive Beeinflussung einer Person, indem Möglichkeiten für Bildungsprozesse bzw. Lernsituationen bereitgestellt werden (Giesecke 1997, 22ff.). Handeln ist dann pädagogisch dadurch, 
«dass dem Lernenden Themen angeboten, auch aufgedrungen werden, um über die Zeit Zustände von Personen zu treffen, sie zu ändern und sie damit zu befähigen, einigermassen selbständig mit den Anforderungen zurechtzukommen, auf die sie in ihrem Leben treffen» (Giesecke 1997, 17).

In der Funktion des McQuailschen Mobilisierens finden sich beispielsweise Analogien zu der von Giesecke als Animieren beschriebenen Grundform pädagogischen Handelns. Das Animieren ziele dabei auf die Initiierung von Lernprozessen in einer gegebenen Situation. Pädagogische Fachkräfte bewegen andere Personen dazu, sich auf Lernchancen einzulassen und diese für sich zu nutzen (Giesecke 1997, 105ff.). Die aktive Herstellung von Zugehörigkeit als eine Unterfunktion der Expression gilt als wichtige pädagogische Handlung in der OKJA. Cloos et al. sehen in der Herstellung von Zugehörigkeit zum sozialen Raum der Jugendarbeit die Vorstufe für die Etablierung einer Arbeitsbeziehung zur pädagogischen Fachkraft (Cloos et al. 2009, 18).

Die Funktion des Interpretierens relevanter Informationen beschreiben Mishna et al. ebenfalls als beziehungsbeeinflussendes Element. Informationen, die pädagogischen Fachkräfte per E-Mail zugesandt bekommen, können diese in der späteren Beratungssituation gewinnbringend nutzen (Mishna et al. 2012, 280f.).

Auf die wichtige Funktion der Informationsdistribution und des Informationserhalts für die pädagogischen Fachkräfte wurde bereits hingewiesen. Aus diesen vorherigen, theoretischen Überlegungen ergibt sich nun folgende, empirisch an der Praxis zu überprüfende Forschungsfrage:

Welche Funktionen schreiben pädagogische Fachkräfte SMP zu und inwiefern nehmen sie SMP als pädagogische Werkzeuge wahr?

Diese Frage wird in zwei Beiträgen verfolgt und beantwortet. Zuerst wird analysiert, welche Funktionen die pädagogischen Fachkräfte der Nutzung von SMP zuschreiben. Im zweiten Beitrag, der in einer Fachzeitschrift für in der Praxis tätige Fachkräfte erschienen ist, geht es um die Vorbildfunktion in der Nutzung von SMP von Seiten der pädagogischen Fachkräfte für junge Menschen.

In dem Beitrag «Mehr als Öffentlichkeitsarbeit» werden wissenschaftliche Publikationen und Erfahrungsberichte analysiert und eine Dysbalance von «informativselbstdarstellend» und «sozial-interaktiv» geschilderten Tätigkeiten (Alfert 2013, 91) identifiziert. Den «informativ-selbstdarstellenden» Tätigkeiten, beispielsweise zum Zweck der Öffentlichkeitsarbeit, wird in der Praxis immenses Gewicht zugeschrieben. Um dieses Übergewicht empirisch zu überprüfen, werden Erkenntnisse der bereits erwähnten Untersuchung von Cloos et al. (2009) als Deskriptionsfolie herangezogen. Anhand der Folie werden exemplarische Situationen der SMP-Nutzung eines Jugendarbeiters pädagogisch beschrieben. Die Dysbalance stellt sich vor dieser Folie als oberflächliche Schilderung heraus. Ein grosser, jedoch zunächst implizit bleibender, 
pädagogischer Gehalt in der SMP-Nutzung durch den Jugendarbeiter tritt auf Grund der genauen Analyse deutlich zu Tage:

Im Hinblick auf die oben genannten Punkte, aber insbesondere im Hinblick auf den Aufbau von Arbeitsbeziehungen zwischen pädagogischen Fachkräften und jungen Menschen hat die Nutzung von SMP pädagogischen Gehalt. Dies zeigt sich beispielsweise darin, dass SMP-Accounts bewusst angelegt und deren Funktionen genutzt werden, um den jungen Menschen einen niedrigschwelligen Zugang zur JFE zu ermöglichen. Ebenso dient die Dokumentation von Ereignissen in der JFE der Stärkung des Zugehörigkeitsgefühls der jungen Menschen zu der JFE. Ursachen für die Übergewichtung durch die pädagogischen Fachkräfte kann der Beitrag nur vermuten. Beispielsweise wäre es möglich, dass auf Grund fehlender pädagogischer Begrifflichkeiten auf bekannte Begriffe aus dem Marketing zurückgegriffen wird.

Für die jungen Menschen können pädagogische Fachkräfte in ihrer Mediennutzung Vorbilder sein, denn auch diese funktionalisieren SMP für sich und sehen darin eine grosse Ressource (Stix und Harneid 2019). Junge Menschen nutzen SMP zur Kontaktpflege und Vernetzung sowie zur Anbahnung von Kontakten (am Beispiel Chatten: Kammerl 2006). Eine Studie des JFF zeigte ausserdem die Bedeutung von SMP bei der Entwicklung einer eigenen Identität (Wagner et al. 2013). Damit im Zusammenhang stehen Funktionen der Selbstdarstellung und die Präsentation von Kreativprojekten (Wagner et al. 2009). Schmidt und Taddicken kommen zu der zusammenfassenden Erkenntnis, dass es vor allem drei Praktiken gibt: das Identitätsmanagement, das Beziehungsmanagement und das Informationsmanagement (Schmidt/Thaddicken 2017). In Anbetracht einer Anzahl spezifischer Herausforderungen, die sich jedoch bei der Nutzung von SMP für die Nutzenden ergeben ${ }^{6}$, können pädagogische Fachkräfte die jungen Menschen bei deren Bewältigung unterstützen.

In der Publikation mit dem Titel «Gute Vorbilder für die Medienkompetenz» wird daher die bedeutsame Funktion pädagogischer Fachkräfte als Vorbilder für das Medienhandeln junger Menschen empirisch begründet dargestellt. Wenngleich in diesem Beitrag kein Bezug zu theoretischen Modellen vorgenommen wird, stellen die

6 Die von den jungen Menschen und pädagogischen Fachkräften hauptsächlich genutzten SMP Facebook und WhatsApp sind im Hinblick auf Verbraucherschutz und Datenschutz sowie hinsichtlich des Jugendschutzes nicht unumstritten (vgl. Mansholt 2018; Wilkens, 2009a,b; mpfs 2012, 44). In jüngster Zeit ist eine stärkere Aufmerksamkeit für die psychsozialen Risiken hinzugekommen. Hinter der - wie junge Menschen sagen «Internetsucht» und «Social-Media-Krankheit» - stehen ernsthafte Krankheiten wie Abhängigkeit, Depression, Angststörungen und Burnout subsumiert, die durch die Nutzung von SMP entstehen oder befördert werden. Forschende sehen junge Menschen als besonders anfällig dafür an, da sie sich in einer kritischen und potenziell anfälligen Phase der sozialen und emotionalen Entwicklung befinden (DIVSI 2018; RSPH 2017; DAK 2017). Weitere identifizierte Faktoren, die zu einer Abnahme der Lebensqualität/-zufriedenheit junger Menschen durch die Nutzung von SMP führen, sind Schlafmangel (RSPH 2017, 17ff.; DAK 2017, 18), verschlechtertes Körperbild und fragiles Selbstbewusstsein (RSPH 2017, 17ff.). Die Forschenden schliessen daraus, dass sich der Körperkult im Internet negativ auf das fragile jugendliche Selbstbewusstsein auswirkt. Auch die Angst vor Beleidigungen (DIVSI 2018, 26 u. 66; MPFS 2018,63 ) und die Angst, etwas zu verpassen (FOMO = Fear of Missing Out) (RSPH 2017, 17ff.) wurden als Faktoren identifiziert. 
«vier Dimensionen der Lernchancen» (Giesecke 1980, 163ff.) ein sensibilisierendes Konzept bei der Analyse und Deskription dar.

1. Die lebensbegleitende Dimension: Aus ihrer Lebensgeschichte, ihren Interessen und Befindlichkeiten heraus bestimmen die jungen Menschen die Inhalte der pädagogischen Kommunikation mit.

2. Die korrigierende Dimension: Wenn Sozialisationsergebnisse korrrigiert und Identität neu erarbeitet werden müssen, kann die Jugendarbeit dazu dienen, Normen, Einstellungen und Verhaltensweisen zu vergleichen, neue Sozialisationserfahrungen zu machen sowie Bedürfnisse und Interessen zu entwickeln.

3. Die aktuelle Dimension: «Aktuell [ist] das, was in der relativ offenen pädagogischen Kommunikation in der Jugendarbeit zur Geltung und zur Sprache kommt. Insofern enthält diese Dimension eine Fülle kreativer und innovativer Möglichkeiten» (Giesecke 1980, 165).

4. Die solidarisierende Dimension: Hier wird das Bedürfnis aufgegriffen, in Peergroups zusammen zu sein und zu erfahren, dass als individuell erfahrene Probleme und Konflikte kollektive sind.

In den genannten vier Dimensionen können die pädagogischen Fachkräfte als Vorbilder dienen und Lernchancen eröffnen: Sie können die jungen Menschen anregen ihr Medienhandeln zu reflektieren. Sie können Vorbilder dafür sein, wie man mit Medieninhalten umgeht, die einen selbst oder andere gefährden, und sie können vorleben, wie man sich datensensibel im Internet bewegt. Nicht zuletzt können die pädagogischen Fachkräfte Praktiken aufzeigen, dem Gruppendruck und der Angst, etwas zu verpassen (FoMo), zu begegnen.

\subsection{Social-Media-Plattformen als pädagogische Handlungsräume}

Wie die Analyse der Datenlage gezeigt hat, gibt es neben der Möglichkeit, SMP funktional als Werkzeuge zu betrachten, auch den (überwiegend theoretischen) Ansatz, SMP als Räume pädagogischen Handelns zu betrachten und sozialräumliche Konzepte potenziell auf die (pädagogische) Arbeit mit SMP zu übertragen (vgl. Gerodetti 2009).

Dieser Ansatz basiert auf dem von Löw 2001 entwickelten raumsoziologischen Konzept des «relationalen Raummodells». Die Grundidee des Konzepts ist, dass Menschen Räume performativ durch ihr Handeln und ihre Interaktionen herstellen. Der Kern ihres Modells besteht darin, einen Raum nicht länger als konkreten Ort oder als "Container», als einen physischen Raum mit vier Wänden und einer Decke zu betrachten, sondern als ein durch Handeln hergestellter Raum. Dies wird auch im alltäglichen Sprachgebrauch an Begriffen wie Sozialraum, öffentlicher Raum oder virtueller Raum sichtbar. Räume sind also nach Löw ein Ergebnis von Handlungen 
- Handeln strukturiert Räume. Gleichzeitig strukturieren Räume Handlungen, was bedeutet, dass Räume Handlungen sowohl einschränken als auch ermöglichen können (Löw 2001, 172).

Implizit findet sich bereits bei Giesecke die Idee, dass Räume performativ, durch wechselseitige Interaktionen, hergestellt werden. Für ihn ist eine Einrichtung wie ein Jugendclub per Definition eine pädagogische Institution, aber nicht alle Handlungen von pädagogischen Fachkräften sind per se pädagogisch. Umgekehrt mache jedes pädagogische Handeln einen Raum zu einem pädagogischen. Die bereits erwähnten Autoren Cloos et al. (2009) gehen ebenfalls davon aus, dass die pädagogischen Fachkräfte durch ihr Handeln und ihre Interaktionen mit den jungen Menschen pädagogische Räume herstellen?

Professionelles pädagogisches Handeln findet laut Giesecke stets in Institutionen statt, in denen es Lernfelder gibt, die ihrerseits Lernsituationen enthalten (Giesecke 1997, 47): (1) Institutionen wie die Jugendarbeit oder Schule ermöglichen planbares und fortschreitendes pädagogisches Handeln und schützen vor falschen Erwartungen. (2) Pädagogische Felder definiert Giesecke als dauerhafte soziale Kontexte, die wechselseitiges, unmittelbares Handeln möglich machen (Giesecke 1997, 55). (3) Pädagogische Situationen stellen für Giesecke ein persönliches Umfeld dar, in dem eine wechselseitige, direkte Interaktion möglich ist. Pädagogisches Handeln ist laut Giesecke immer interaktives, soziales Handeln, da es auf das Handeln anderer Menschen bezogen und vom Handeln des Gegenübers beeinflusst ist (Giesecke 1997, 21f.).

Davon ausgehend entwickelt er die bereits auszugsweise erwähnten fünf Grundformen pädagogischen Handelns. In ihrer Summe ergeben sie laut Giesecke die professionelle Kompetenz des pädagogischen Berufs (Giesecke 1997, 76ff.)

Unterrichten: Pädagogische Fachkräfte erklären bzw. referieren relativ komplexe Sachzusammenhänge in einem längeren Argumentationsprozess (Giesecke 1997, 79).

Informieren: ist für Giesecke immer auf aktuelle Lebenssituationen bezogen. Es erfolgt im Alltagsleben und dient dazu, jemanden von einer Verhaltensunsicherheit zu befreien (Giesecke 1997, 84).

Beraten: Die Beratung setzt bei der pädagogischen Fachkraft fundierte Kenntnisse über den Gegenstand voraus und ist die diskreteste und

7 Die Autoren entwickeln für die Räume den Begriff «pädagogische Arena» (Closs et al. 2009).

8 Es ist wichtig zu erwähnen, dass Giesecke zum damaligen Zeitpunkt die Informationsausgabe von Massenmedien (gemeint sind Radio, TV, Presseerzeugnisse) von jeglicher Art pädagogischen Handelns unterscheidet. Er macht geltend, dass das pädagogische Handeln immer in einem Umfeld stattfindet, in dem eine wechselseitige, direkte Interaktion möglich ist (Giesecke 1997, 47ff.). Lediglich nach der Rezeption von Massenmedien könne, durch diese angeregt, eine pädagogische Situation entstehen. Da die Interaktion der Nutzenden untereinander ein entscheidendes Definitionsmerkmal des sogenannten Web 2.0 im Allgemeinen und der hier behandelten SMP im Besonderen ist, ist Gieseckes veralteter Standpunkt zu vernachlässigen. 
Arrangieren: Wenn pädagogische Fachkräfte Gelegenheiten für offene Lernprozesse arrangieren, schaffen sie eine Situation, «die selbst erzieherisch wirkt» (Giesecke 1997, 96). Pädagogische Fachkräfte arrangieren in den verschiedensten Situationen, beispielsweise um Diskretion zu gewährleisten und Störungen zu vermeiden, oder wenn sie sich selbst als Ansprechpartner_in und somit als Lernmöglichkeit zur Verfügung halten. Giesecke weist darauf hin, dass nicht alles, was eine pädagogische Fachkraft tut, automatisch pädagogisches Handeln ist. Ein Lagerfeuer könne beispielsweise primär der Unterhaltung dienen, gleichwohl es dem pädagogischen Klima vermutlich förderlich ist (Giesecke 1997, 101).

Animieren: zielt auf die Initiierung von Lernprozessen in einer gegebenen Situation. Pädagogische Fachkräfte bewegen andere Personen dazu, sich auf Lernchancen einzulassen und diese zu nutzen. Animieren impliziert auch ein Ausprobieren und Korrigieren der Animationsmittel. Der Erfolg der Animation hängt von der Persönlichkeit der pädagogischen Fachkräfte ab und deren glaubwürdiger Vermittlung der eigenen Interessen (Giesecke 1997, 105ff.).

SMP sind per Definition keine pädagogischen Institutionen. Da in/auf ihnen aber pädagogisches Handeln stattfindet, liegen, ausgehend von den obigen Ausführungen zu performativ hergestellten Räumen, die Überlegungen nahe, SMP als pädagogische Handlungsräume zu betrachten und mittels dieser Perspektive das pädagogische Handeln der Fachkräfte in/auf SMP aufzuschlüsseln und implizite Handlungsstrukturen aufzudecken. Für die vorliegende Arbeit ergibt sich demnach die folgende Frage. Inwiefern können SMP pädagogische Handlungsräume darstellen und inwiefern bergen SMP dann Potenziale für eine professionell-pädagogische Nutzung durch pädagogische Fachkräfte?

Die Publikation «Social Media Platforms as Pedagogical Spaces» geht dieser Frage nach $^{9}$. Es wird aufgezeigt, dass viele alltägliche Situationen in der Nutzung von SMP in der OKJA pädagogische Situationen enthalten. Die Grundformen pädagogischen Handelns, Arrangieren, Animieren, Informieren und Beraten finden sich in vielfachen Beispielen in der Nutzung von SMP durch die pädagogischen Fachkräfte und werden in den Beiträgen analytisch dargelegt. Lediglich die Form des Unterrichtens konnte

9 Eine deutschsprachige, allerdings stark gekürzte Fassung mit dem Titel «Soziale Onlinenetzwerke als pädagogische Handlungsräume» wurde 2018 in Sozial Extra (Jg. 42, Nr. 3, S. 157-168) veröffentlicht. 
im pädagogischen Handeln der pädagogischen Fachkräfte nicht identifiziert werden, was angesichts des Handlungsfelds OKJA auch nachvollziehbar ist. Es wurde deutlich, dass die Einordnung von SMP als Werkzeuge der pädagogischen Fachkräfte zu kurz greift und es sowohl für den theoretischen Diskurs als auch die Argumentation in der Praxis förderlicher ist, SMP als pädagogische Handlungsräume zu betrachten. Die sich daraus ergebenden ethischen und datenschutzrechtlichen Schwierigkeiten werden als besonders zu reflektierenden Herausforderungen für die pädagogischen Fachkräfte identifiziert. Sich mit ihnen auseinander zu setzen ist ein zentraler Punkt im weiteren Diskurs zur Professionalisierung der Nutzung von SMP durch pädagogische Fachkräfte.

\subsection{Professionalität im Medienhandeln}

Mishna et al. (2012) kommen in einer Untersuchung zu der Erkenntnis, dass inzwischen entscheidende - und mehr als zunächst bewusste oder angenommene - Kernelemente des professionellen Handelns durch Informations- und Kommunikationstechnologien (im Folgenden kurz IKT) beeinflusst werden ${ }^{10}$. Sie identifizieren einen Prozess des Einschleichens, den sie als Creep-In bezeichnen (Mishna et al. 2012, 280). Beispielsweise würden E-Mail-Kontakte nicht nur zur vereinbarten Terminverschiebung verwendet, sondern zusätzlich genutzt, um Gesprächsinhalte anzukündigen (Mishna et al. 2012, 280f.). Diese Onlinekommunikation ist kein geplanter Bestandteil der sozialarbeiterischen Intervention, sie wird jedoch unmittelbar Teil des Interventionsprozesses und dementsprechend bedeutsam (Mishna et al. 2012, 278). Zugleich stellt dies einen Grenzübertritt dar und wirft die Frage auf, ob und inwiefern es vertretbar ist, solche Kontaktmöglichkeiten wieder rückgängig zu machen und Grenzen wiederherzustellen. Die befragten pädagogischen Fachkräfte befürchten beispielsweise bereits, dass ihre Klientinnen und Klienten zeitverzögertes Antworten als mangelndes Interesse oder Engagement fehlinterpretieren (Mishna et al. 2012, 281). Auch die zunehmende Erwartungshaltung einer Rund-um-die-Uhr-Erreichbarkeit führt bei den pädagogischen Fachkräften zu dem Bedürfnis, klare professionelle Grenzen (wie Erreichbarkeit/Bürozeiten, Rollenverständnis und Verantwortlichkeiten) zu kommunizieren.

10 In der Untersuchung wurden fünfzehn nordamerikanische Sozialarbeiterinnen und Sozialarbeiter mittels Fokusgruppen- oder Tiefeninterviews befragt. Sie wurden durch E-Mail-Verteiler über Berufsverbände und Alumni-Vereine rekrutiert und erfüllten diverse Teilnahmevoraussetzungen (Abschluss als Social Worker, staatliche Anerkennung, aktuelles Beschäftigungsverhältnis in öffentlichem Sozialdienst oder Tätigkeit in Privatpraxis, lokale Nähe) Mishna et al. 2012, 279). Die Datenanalyse erfolgte mittels Grounded-Theory-Methodologie nach Strauss/Corbin (Mishna et al. 2012, 280). Von den 15 pädagogischen Fachkräften, die an der Studie teilnahmen, waren 13 weiblich und zwei männlich. Sie waren im Alter von Mitte 30 bis Ende 50 und hatten mindestens einen Master of Social Work. Alle hatten mehrjährige Berufserfahrung und arbeiteten in traditionellen Face-to-Face-Beratungssettings, die meisten in Sozialdiensten. Die Handlungsfelder umfassten (psychische) Gesundheit, Bildung sowie Kinder- und Familienservice (Mishna et al. 2012, 279). 
Neben den bisher genannten «Grenzaufweichungen und -übertritten», die neue «ethisch-professionelle Fragen» aufwerfen, identifizierten Mishna et al. unter anderem das Phänomen, dass Onlinekommunikation mit einem legereren sprachlichen Ausdruck einhergeht (Mishna et al. 2012, 282), der auch in die Face-to-Face-Settings einfliesst. Des Weiteren thematisierten sie ein Dilemma auf Seiten der pädagogischen Fachkräfte, da sie durchaus auch Vorteile in der Onlinekommunikation sehen, aber das Vertraulichkeits- und Verschwiegenheitsprinzip bei der Onlinekommunikation nicht garantieren können (Mishna et al. 2012, 281f.).

Entscheidend sei, dass im Gegensatz zur Onlineberatung, für welche seit längerem Regeln und Richtlinien existieren, die Nutzung von IKT im Kontext traditioneller Face-to-Face-Beratung noch eine individuelle Angelegenheit ohne praktische und ethische Leitlinien sei (Mishna et al. 2012, 278). Letztlich läge, so Mishna et al., die Entscheidung, ob traditionelle Settings der sozialpädagogischen Arbeit von Internetkommunikation beeinflusst werden, nicht mehr in den Händen der pädagogischen Fachkräfte. IKT würden sich, unabhängig von den persönlichen und fachlichen Präferenzen der pädagogischen Fachkräfte und der Träger, weiter in die professionellen Settings der Sozialen Arbeit einschleichen, da Onlinekommunikation alltäglich geworden und Teil eines unumkehrbaren gesellschaftlichen Prozesses sei. Ein Gedanke, der an früherer Stelle in dieser Arbeit zu Krotz' Mediatisierungstheorie bereits dargelegt wurde.

Die Arbeits- und Techniksoziologin Carstensen greift die Thematik der Grenzüberschreitung aus zwei weiteren Perspektiven auf (2015). Sie untersuchte, inwiefern die Mediatisierung der Arbeitswelt und insbesondere die Durchsetzung von mobilen IKT zu Belastungen und Stress führen. Es geht also nicht, wie oben, um eine Grenzüberschreitung durch eine andere handelnde Person, sondern es geht einerseits darum, dass gewisse technische Entwicklungen eigene grenzüberschreitende Handlungen ermöglichen, und andererseits um die gesundheitlichen Konsequenzen, die sich aus Grenzüberschreitungen ergeben.

Mobile IKT befördern laut Carstensen die Grundlage für eine räumliche und zeitliche Entgrenzung des Beruflichen, da sie unter anderem eine permanente Erreichbarkeit ermöglichen (Carstensen 2015, 187). Die Nutzung privater Geräte für berufliche Zwecke oder beruflicher Geräte für private Zwecke verschärfe sogar diese Entgrenzung, da Grenzen mit einem Klick überschritten werden können. Carstensen spricht in diesem Zusammenhang den von Mishna et al. (2012) ebenfalls thematisierten schleichenden Prozess an:

«Hierbei geht es meistens nicht mehr wie bei Telearbeit um eine bewusste Entscheidung statt im Büro zu Hause zu arbeiten, sondern um eine schleichende Entwicklung, die permanentes Arbeiten via Smartphone und Laptop zu allen Zeiten und an allen Orten zunehmend ermöglicht» (Carstensen 2015, 189). 
Des Weiteren zeigt sie auf, dass diesbezüglich auch die Erwartungen, permanent erreichbar zu sein, angestiegen sind.

Im Hinblick auf arbeitsschutzrechtliche Aspekte argumentiert sie, dass jegliches Abrufen und Lesen einer Nachricht mit beruflichem Inhalt als Arbeitszeit zu werten sei. Geschehe dies in der Freizeit, könne das mit den im Arbeitszeitgesetz vorgeschriebenen und für die Erholung wichtigen Ruhezeiten, die grundsätzlich elf Stunden ohne Unterbrechung betragen, kollidieren (Carstensen 2015, 189). Ohne Frage ermögliche der Einsatz von mobilen (eigenen) Geräten auch grössere Autonomie (vgl. Carstensen 2015, 188) oder bringe Informiertheit ein entlastendes Gefühl (vgl. Carstensen 2015, 189). Jedoch berge die Technik - in Verbindung mit in einem Wechselverhältnis stehenden gesellschaftlichen Verhältnissen - auch ein Belastungspotenzial. Laut Carstensen stehen «psychische Belastungen, Stress und Burn-Out zweifelsohne im Zusammenhang mit den gewandelten Arbeitsbedingungen» (Carstensen 2015, 187).

Die genannten Aspekte beeinflussen das pädagogische Handeln und müssen daher im Rahmen einer professionellen SMP-Nutzung von pädagogischen Fachkräften reflektiert werden. Der Wunsch, dies empirisch zu überprüfen, führt zu folgender Forschungsfrage:

Welche Strategien, SMP professionell zu nutzen, finden sich im pädagogischen Handeln auf und mit SMP in der OKJA?

Diese Frage wird in der Publikation «Schleichende Grenzaufweichungen und persönliche Verflechtungen» verfolgt: Eine Erkenntnis ist, dass sich die Handlungsfacetten einer pädagogischen Social-Media-Nutzung bei den untersuchten pädagogischen Fachkräften ausdehnen und es, unter anderem durch die Nutzung privater Mobilgeräte, zu Grenzaufweichungen und persönlichen Verflechtungen kommt. Dies sowie die Tatsache, dass sich die jungen Menschen ständig neuen SMP zuwenden, erfordert eine stetige und reflektierte Anpassung der Handlungsstrategien, so eine Schlussfolgerung. Ausgehend von Fallbeispielen wurden Handlungsprämissen entwickelt. Diese sind als rahmende Voraussetzung für professionelles pädagogisches Handeln zu verstehen und können des Weiteren sensibilisierend als Grundlage für Reflexion des professionellen Handelns oder der Entwicklung einer Social-MediaPolicy dienen. Die Handlungsprämissen lauten: pädagogenfreie Zonen, Nachrangigkeitsgrundsatz, kritische Offenheit als Haltung, klare Zielformulierungen, Reflexionen und pädagogische Grenzen. In die Formulierung dieser Handlungsprämissen sind sämtliche Erkenntnisse, die im Rahmen der vorliegenden Arbeit empirisch und theoretisch gewonnen wurden, eingeflossen.

Die zentralen Ergebnisse der gesamten Forschungsarbeit lassen sich übergeordnet drei Themenfeldern zuordnen 1) strukturelle Bedingungen der OKJA, 2) Herausforderungen für die Professionalisierung und 3) pädagogisches Handeln in 
pädagogischen Räumen. Sie werden in "Forschungsergebnisse und Schlussfolgerungen - Manteltext Schlussteil» ausführlich dargelegt und dienen als Basis für die Ableitung von Konsequenzen für pädagogisches Handeln sowie die Benennung von Forschungsdesideraten.

Mit der Beantwortung der Forschungsfragen bereichert die vorliegende Arbeit den wissenschaftlichen Diskurs der Sozialen Arbeit im Allgemeinen und der OKJA im Speziellen um Erkenntnisse zur professionellen, pädagogischen Nutzung von SMP. Als praxisorientierte Forschung wurden die Handlungsmuster der pädagogischen Fachkräfte sowie deren Binnenlogiken und Rahmenbedingungen erfasst und rekonstruiert. Die Arbeit bietet damit erstmals einen differenzierten Überblick und eine systematische Deskription der offensichtlichen und weniger offensichtlichen Bedingungen (vgl. Creep-in-Phänomen) professionell, insitutionellen Handelns von pädagogischen Fachkräften der OKJA im Kontext von SMP. Die Erkenntnisse der Arbeit können somit den Professionalisierungsdiskurs bereichern und als Ausgangsbasis für weitere Theoriebildung, Beobachtung, Reflexion und Weiterentwicklung von Disziplin und Profession herangezogen werden.

Insofern ergänzt die vorliegende Arbeit nicht nur den wissenschaftlichen Diskurs, beispielsweise indem empirisch aufgezeigt wird, dass ein performatives Raumverständnis das pädagogische Handeln der Fachkräfte in/auf SMP aufzuschlüsseln vermag, und indem implizite Handlungsstrukturen aufgedeckt werden. Im Rahmen der Arbeit wurde zudem Wissen generiert, das Anschlusspotenziale für Praxis und Administration bietet. Beispielsweise bieten die Erkenntnisse eine konzeptionellinhaltliche Orientierung für die pädagogische Nutzung von SMP in der OKJA und ermöglichen somit eine methodisch geleitete Nutzung. Des Weiteren können die Ergebnisse eine Grundlage für die Durchsetzung angemessener Prämissen im Sinne von Rahmenbedingungen professionellen Handelns und für eine administrative Entscheidungsfindung darstellen.

Die in diesem Kapitel aufgezeigten Entwicklungen von Forschungsfoki und Forschungszielen waren sinngemäss eng mit der empirischen Untersuchung im Allgemeinen und dem Forschungsstil der Grounded Theory im Besonderen verknüpft. Die empirische Untersuchung und ihre methodische Umsetzung wird nun im Folgenden erörtert.

\section{Empirische Untersuchung}

Um das Medienhandeln und die Handlungsmotive pädagogischer Fachkräfte sowie dessen Rahmenbedingungen zu beschreiben und rekonstruieren erschien für die vorliegende Untersuchung ein qualitatives Forschungsdesign angemessen (Flick 2002, 16). Durch eine strukturelle Offenheit und Flexibilität im Forschungsprozess ermöglichte das qualitative Vorgehen sowohl antizipierte Phänomene zu explorieren, 
liess dabei aber zugleich nicht-antizipierten Phänomenen Raum zur Entdeckung. Auch konnten bei dem qualitativen Vorgehen die subjektiven Erfahrungen, Sichtweisen und Schwerpunktsetzungen der pädagogischen Fachkräfte in die Untersuchung einbezogen und berücksichtigt werden.

Die zentralen methodischen Prinzipien qualitativer Sozialforschung (Offenheit, Forschung als Kommunikation, Prozesscharakter, Flexibilität und insbesondere die Reflexivität von Gegenstand und Analyse) (vgl. Lamneck 2005, 20f.) erschienen für das geplante explorative Forschungsprojekt grundlegend.

\subsection{Reflexive Grounded Theory als Forschungsstil}

Die Reflexive Grounded Theory nach Breuer (2010) ist eine die Subjektivität und Selbstreflexivität in den Fokus rückende "Lesart» (Mey und Mruck 2011, 21) der ursprünglichen Grounded-Theory-Methodologie (Glaser und Strauss 1967/2006). Sie kann unter anderem als Methodenset der Datenanalyse betrachtet werden (siehe methodisches Design der Datenauswertung) ebenso wie als ein den Forschungsprozess umfassenden Forschungsstil (vgl. Mey und Mruck 2011, 22; Breuer et al. 2011; Breuer et al. 2010, 39ff. und 51ff.). Eine solche Orientierung an Letzerem und insbesondere an den reflexiven Anteilen erscheint aus diversen Gründen relevant und notwendig für die vorliegende Arbeit:

Laut Breuer eignet sich die Methodik der Reflexiven Grounded Theory «speziell für sozialwissenschaftliche Untersuchungsanliegen [...], die auch Züge «persönlicher Projekte> besitzen» (Breuer et al. 2010, 10, vgl. ebenso Charmaz 2011, 187). Meine persönliche Nähe zum Untersuchungsgegenstand und Feld ist in diesem Fall der ausschlaggebende Faktor. Zwar könne man bei jedem lebensweltbezogenen Forschungsthema "gewisse Berührungspunkte [...] und damit zusammenhängende emotional-affektive Reaktionen» (Breuer et al. 2011, 431) annehmen, doch in meinem Fall gibt es vielfältige berufliche Bezüge, die eine entsprechend kontinuierliche Reflexion elementar erscheinen lassen.

Geeignet erscheint die Reflexive Grounded Theory auch, weil sie Forschende in besonderem Masse anhält, ebenso auf liminale Bedeutungen bzw. implizite Handlungen wie auf explizite Aussagen und Handlungsweisen zu achten (vgl. Charmaz 2011, 195 sowie Breuer et al. 2010, 50). Um dies zu unterstützen, wurden als Methode der Datenerhebung episodische Interviews gewählt.

\subsection{Methodisches Design der Datenerhebung: Episodische Interviews}

Der Grundgedanke der episodischen Interviews basiert auf den Erkenntnissen der Gedächtnis- und Wissenspsychologie, demzufolge Menschen über verschiedene Wissensspeicher verfügen: episodisch-narratives und semantisch-begriffliches Wissen. 
Das episodische Gedächtnis speichert und verarbeitet autobiografische Informationen, also solche Informationen, die auf eigenen Erfahrungen basieren. Das episodische Wissen umfasst kontextuell gebundenes Wissen. Solch erfahrungsnahes Wissen ist mit der Erinnerung an konkrete Situationen verbunden. Daneben gibt es das semantische Wissen. Dies umfasst generalisiertes Wissen wie Regeln, Definitionen, abstrahierte Annahmen und Zusammenhänge, die nicht an einen bestimmten Kontext gebunden sind (Flick 2011b, 28ff.). Informationen zum episodisch-narrativen Wissen eines Menschen können Forschende über Erzählungen, die eine kontextsensitive Ebene ansprechen, aufdecken. Informationen zum semantisch-begrifflichen Wissen eines Menschen erhält man durch argumentativ-theoretische Erörterungen der/des Interviewten. Episodische Interviews stellen somit eine methodeninterne Triangulation dar, die systematisch verschiedene Interviewstile kombiniert (Flick 2002, 160). Dass dadurch automatisch verschiedene Datensorten generiert werden, sieht Flick als Chance, unterschiedliche Zugänge zu den Sicht- und Erfahrungsweisen der Interviewten zu ermöglichen (Flick 2011a, 279).

Vor dem Hintergrund der Erkenntnisse von Knoll-Krist (1985) sind diese unterschiedlichen Zugänge bedeutsam (Knoll-Krist 1985, 24-26): Knoll-Krist stellte fest, dass die interviewten pädagogischen Fachkräfte grosse Schwierigkeiten hatten, Ereignisse ohne Interpretation darzustellen. Ihm fiel zudem auf, dass eine starke Tendenz zur Abstraktion und Theoriebildung vorliegt. Des Weiteren berichtet er, dass es den pädagogischen Fachkräften schwer gefallen sei zu objektivieren, wenn es um das Selbst ging. Erst auf Nachfrage erhielt er subjektive Darstellungen. Diese Ambivalenz spiegele sich auch in der Sprache wider, diese sei eine Mischung aus wissenschaftlicher Darstellung und Jugendsprache. Sie enthielte neben relativer Wissenschaftlichkeit auch Unterstellungen und Implikationen, wie sie der Umgangssprache eigen sind. Vertiefte Darstellungen seien «oft erst auf der Basis der konkreten Schilderung möglich», schlussfolgert Knoll-Krist deshalb (Knoll-Krist 1985, 122). Um die vertieften Darstellungen der pädagogischen Fachkräfte zu erhalten, eignen sich also die narrativen Anteile episodischer Interviews, während die definitorischen Elemente die semantischen Anteile episodischer Interviews abdecken. Dies ist auch unter Berücksichtigung der Erkenntnisse von Thole et al. wichtig, da diese eine grosse Spannweite «sehr individueller Definitionen» identifizierten, die vom Fachdiskurs entfernt bzw. eine ungenaue Anlehnung seien (Thole und Küster-Schapfl 1997, 58). Die Praxis basiere auf eigenem Erfahrungs- und Wissensnetz und sei «nicht einmal ansatzweise kompatibel» mit dem akademisch gelehrten Wissen (Thole und KüsterSchapfl 1997, 218). Daher erschien es notwendig, die individuellen Definitionen und 
Wissensvorräte, die die Basis des pädagogischen Handelns darstellen, mittels methodeninterner Triangulation in Form episodischer Interviews zu erfragen ${ }^{11}$.

Von Vorteil ist des Weiteren, dass die Methode der episodischen Interviews nicht nur bereits in verschiedenen Kontexten der (gesundheitsorientierten) Sozialen Arbeit erprobt, sondern auch aus ihnen heraus entwickelt wurde (Flick 2011a). Laut Flick eignen sich episodische Interviews, a) um Professionelle zu ihrem beruflichen Handeln zu befragen (Flick 2011a, 267), und b) bei Untersuchungen, die «auf Wissen, Erfahrungen und Veränderungen aus der Sicht der Befragten abzielen» (Flick 2011a, 278) und «Routinisierungen und Alltäglichkeiten» zu analysieren versuchen (Flick 2002, 165).

\subsection{Methodisches Design der Datenauswertung: Theoretisches Kodieren nach der (Reflexiven) Grounded Theory}

Steht - wie im Fall der vorliegenden Arbeit - kein Vergleich so genannter gruppenspezifischer Sichtweisen im Fokus, legt Flick das theoretische Kodieren als Auswertungsmethode für episodische Interviews nahe (Flick 2002, 166).

Als Methodenset der Datenanalyse ist das Vorgehen nach der Reflexiven Grounded Theory - von einigen Akzentsetzungen und Erweiterungen abgesehen - mit dem von Strauss/Corbin (1996) sowie Strauss (1991) vergleichbar und daran angelehnt (vgl. Breuer at al. 2010, 40). Strauss benennt als eine Art Methodenset drei «Essentials», die es bei der Anwendung der Grounded-Theory-Methodologie zu berücksichtigen gelte: theoretisches Kodieren, theoretisches Sampling und Vergleiche (Strauss et al. 2011, 75). Daneben betrachtet Strauss den Stil der Grounded Theory als «sehr variabel» (Strauss et al. 2011, 75): Die Methodologie müsse an Fragestellungen und Randbedingungen angepasst werden und insbesondere bei praxisorientierten Fragestellungen seien «entsprechende Abstriche notwendig» (Strauss et al. 2011, 75).

Bei Strauss und Corbin sowie bei Breuer beschreibt der Begriff des Kodierens den Prozess der Datenanalyse im Allgemeinen und den Prozess konzeptioneller Aufbereitung von Daten (engl.: conceptualizing data) im Speziellen (Strauss und Corbin 1996, 43). Dieser Prozess umfasst bei Breuer, der sich an Strauss (1991) und Strauss/Corbin (1996) anlehnt, zwei Kodieroperationen. Diese Kodieroperationen stehen zueinander

11 Der Kritik, dass Struktureigenschaften der Praxis nicht allein über die Analyse von Sprechhandlungen aufzuschliessen sei (vgl. Thole et al. 2011, 115) kann mit Maiwald (2003) begegnet werden. Maiwalds Untersuchungen zur Familienmediation haben gezeigt, dass sich bei Interviews «mit Angehörigen professioneller oder professionsnaher Berufe» durchaus Annahmen über die Struktur der beruflichen Praxis treffen lassen, wenn die Interviews offen geführt würden und die Interviewten die Möglichkeit hätten, die Praxis «fallnah» zu schildern (Maiwald 2003, 176). Er argumentiert damit, dass die «reflexive Evaluation einer konkreten Entscheidungssituation» selbst Teil des professionellen Handelns sei (Maiwald 2003, 154). Maiwald räumt aber auch ein, dass Interviews nur begrenzt das faktische Handeln abbilden können und dass Interviews als alleinige Datengrundlage nur eine Perspektive darstellen, weshalb er rät, bei der (von ihm gewählten Sequenzanalyse, Maiwald 2003, 160ff.) weiteres Datenmaterial zu berücksichtigen (Maiwald 2003, 177). 
in einem hierarchischen Verhältnis, überschneiden sich in der praktischen Arbeit jedoch oft und müssen in der Regel mehrfach iterativ durchlaufen werden.

Die erste Kodieroperation ist das so genannte offene Kodieren, «das Herausgreifen einer Beobachtung, eines Satzes, eines Abschnitts und das Vergeben von Namen für jeden einzelnen darin enthaltenen Vorfall, jede Idee oder jedes Ereignis» (Strauss und Corbin 1996, 45). Die so genannten Kodes sind also Schlüsselwörter oder Begriffsassoziationen zu einzelnen Textstellen (bezeichnet als In-vivo- bzw. theoretische Kodes; Breuer et al. 2010, 77). Während Kodes somit relativ datennah sind, sind Kategorien eine Art Oberbegriff, eine Bündelung von Kodes unter einem Konzept höherer Ordnung und mit höherem Abstraktionsniveau. Die Kategorienbildung stellt einen ersten Schritt in Richtung der zweiten Kodieroperation, dem axialen Kodieren, dar, da sie sich sowohl «datengetrieben, d.h. angeregt und inspiriert durch empirische Phänomene» sowie «kritisch-systematisch, d.h. durch Prüfen der theoretischen Potenz der [...] Kategorien» (Breuer et al. 2010, 73) vollzieht. Breuer benennt in diesem Zusammenhang, angelehnt an Strauss und Corbin, das Fragenstellen, Vergleichen und Dimensionalisieren als heuristische Instrumente (Breuer at al. 2010, 81ff.).

Beim axialen Kodieren geht es darum, die aufgebrochenen Daten wieder in Beziehungen zueinander zu setzen. Die Forscherin entwickelt Systematiken für die Beziehung der einzelnen Kategorien. Das paradigmatische Modell stellt das in der vorliegenden Forschungsarbeit am häufigsten herangezogene Instrument dar. Es ermöglicht, Beziehungen zwischen einem Phänomen, seinen Ursachen und Konsequenzen, seinem Kontext und den dabei von den Beteiligten verwendeten Strategien zu veranschaulichen.

\subsection{Umsetzung der Methoden in der Forschungspraxis}

Im Folgenden werden die Schritte der praktischen Umsetzung der gewählten Forschungsmethoden in der vorliegenden Arbeit detailliert beschrieben. Dies umfasst vorbereitende Schritte wie das qualitative Sampling, die Planung des Feldaufenthalts und die Herstellung des Feldkontakts sowie die Erstellung des Interviewleitfadens. Des Weiteren wird der Ablauf der Datenerhebung, die Aufbereitung und Analyse der Daten ausführlich dargelegt.

\subsubsection{Qualitatives Sampling}

Im Rahmen der bereits zuvor erwähnten Studie von Korfmacher (2011) hatten sich 18 pädagogische Fachkräfte bereit erklärt, mein Dissertationsprojekt zum Thema Social Media und Jugendarbeit mittels eines Interviews zu unterstützen.

Für das vorliegende Forschungsprojekt wurden JFE angelehnt an Lüdtke und Grauer (1973) definiert. Die zu interviewenden Personen sollten folgende Kriterien 
erfüllen: (1) Abschluss als Sozialpädagog_in/Sozialarbeiter_in mit Diplom, Bachelor of Arts/Education oder Master of Arts/Education, (2) festangestellt als pädagogische Fachkraft in der JFE, (3) hauptverantwortlich für die Pflege der Social-MediaPräsenz. Es sollte gewährleistet werden, dass eine Person Auskunft gibt, die sowohl professionell sozialpädagogische Handlungskompetenzen aufweisen kann als auch die meisten Erfahrungen mit der beruflichen Nutzung von SMP gesammelt hat und folglich am besten darüber Auskunft geben kann.

Von dieser Idealvorstellung einer kriteriengeleiteten Auswahl der Interviewpartnerinnen und -partner musste hinsichtlich des ersten Kriteriums teilweise abgewichen werden. Zwar hatten alle der Interviewpartnerinnen und -partner einen pädagogischen/erzieherischen Hintergrund, aber nicht alle hatten ein Studium der Sozialen Arbeit/Sozialpädagogik absolviert. Diese Einschränkung wurde aufgrund des begrenzten Pools an Kontaktdaten zunächst hingenommen und erwies sich im Rahmen der Datenanalyse als in seiner Relevanz überschätzt, da sich keine Unterschiede hinsichtlich der professionell pädagogischen Handlungs- und Reflexionskompetenzen zwischen ausgebildeten und studierten pädagogischen Fachkräften zeigten.

Annäherung an das Feld und Leitfadenerstellung

\subsubsection{Annäherung an das Feld}

Im Rahmen der Annäherung an das Feld wurde ein besonderes Augenmerk auf die Rollendefinition, die Planung des Feldaufenthalts inklusive der Leitfadenerstellung und die Herstellung des Feldkontakts gelegt. Die Rollendefinition umfasste unter anderem Überlegungen zur Preisgabe meines Vorwissens als Sozial- bzw. Medienpädagogin. Die Planung des Feldaufenthalts umfasste die konkrete organisatorische Vorbereitung, wie die Klärung des Erhebungsfokus, das Anfertigen von Notizen, Überlegungen zu Zeit/Lokalität sowie zum Fair Return.

Der Interviewleitfaden der ersten Datenerhebungsphase 2012/13 enthielt entsprechend einer Methode von Kruse (2011) verschiedene Frageblöcke zu den Themen persönlicher Erstkontakt mit SONW, Motivation der Anmeldung, erfahrene Reaktionen der Besucherinnen und Besucher, pädagogische Arbeit allgemein und pädagogisches Handeln mit und in SONW, Definitionen und einschätzende Reflexion der SONW-Nutzung. Nach den Pre-Tests wurden den Frageblöcken Relevanzen zugewiesen, um bei Zeitmangel entsprechend priorisieren zu können (vgl. Kruse 2011).

Entgegen Flicks Empfehlung, bei episodischen Interviews zunächst das Verständnis zum Untersuchungsgegenstand zu erfragen (Flick 2011b), habe ich bewusst mit der konfrontativen Frage nach dem ersten Kontakt mit SONW angefangen. Zweck dessen war, dass mir a) sogleich erste Erfahrungen und Emotionen berichtet werden und ich b) en passant erfahre, ob ein Unterschied zwischen beruflicher und privater Nutzung von SONW gemacht wird. Des Weiteren erhoffte ich mir c), dass ein 
grundlegendes Verständnis von SONW bereits bei dieser ersten Einstiegsfrage definiert wird. Die gewählte Einstiegsfrage schätze ich als gelungen ein. Grundsätzlich wurde bei der Ausarbeitung des Leitfadens darauf geachtet, dass die Erzählaufforderungen gegenüber den Fragen überwiegen. In Anlehnung an Witzel (2000) wurde am Ende der Interviews ein Kurzfragebogen zu Person und Einrichtung gemeinsam mit den Interviewpartnerinnen und -partner durchgegangen.

Bei den Interviews im Jahr 2017 lag der Fokus des weniger umfangreichen Leitfadens und des Interviews auf der im Laufe der Zeit veränderten Nutzung von SMP, auf in diesem Zusammenhang entwickelten Strategien und auf dem Einfluss auf die Beziehungen zu den jungen Menschen.

Für die Aufnahme des Feldkontakts wandte ich mich telefonisch an die JFE bzw. an die benannten Ansprechpartnerinnen und -partner aus oben erwähntem Pool. Die Telefonate wurden mit Hilfe eines Telefonleitfadens durchgeführt. Die pädagogischen Fachkräfte reagierten auf meine Anfrage sämtlich sehr offen und - abgesehen von zwei Ausnahmen - erklärten sich alle bereits nach wenigen Sätzen zur Unterstützung bereit, so dass Termine schnell und unkompliziert vereinbart werden konnten. Letzteres traf auch bei der Datenerhebungsphase $2017 \mathrm{zu}$.

\subsubsection{Ablauf der Datenerhebung}

Die zu analysierenden Daten wurden in mehreren Phasen erhoben. Die erste Interviewphase fand 2012/13 statt. In dieser Phase wurden episodische Interviews mit pädagogischen Fachkräften in Einrichtungen der OKJA geführt. Die ersten beiden Interviews galten zunächst als Pre-Tests. Die Interviews dauerten rund 90 Minuten, wobei diese Zeitspanne auch diverse Gesprächsunterbrechungen beinhaltete. Solche Gesprächsunterbrechungen oder auch das spontane Einbeziehen anderer Mitarbeiterinnen und Mitarbeiter oder Besucherinnen und Besucher in die Interviewsituation ggf. verbunden mit dem eigenen kurzen Verschwinden, um etwas zu erledigen, waren kennzeichnend für sämtliche meiner Interviews. Dies verlangte von mir eine hohe Flexibilität in der Interviewführung. Nach den beiden Pre-Tests wurden noch drei weitere episodische Interviews mit insgesamt vier pädagogischen Fachkräften und mit etwas zeitlichem Abstand noch fünf weitere Interviews mit sechs pädagogischen Fachkräften geführt. Sämtliche Interviews fanden in den JFE der pädagogischen Fachkräfte bzw. auf deren Aussengelände statt. Alle Interviewten nahmen freiwillig teil, wobei ein Interviewpartner kurzfristig für seine erkrankte Kollegin einsprang. Von den zehn geführten Interviews wurden zwei spontan im Team von zwei pädagogischen Fachkräften (1x männlich-männlich, 1x männlich-weiblich) durchgeführt. Bei den restlichen Einzelinterviews waren drei der Interviewten weiblich, fünf männlich. 
Im Rahmen der Erhebung im Frühjahr 2017 konnte ein erneuter Kontakt zu sieben der vorherigen Interviewten hergestellt und mit sechs von ihnen Interviews realisiert werden. Die Interviews dauerten rund 45 Minuten und fanden wieder in den Einrichtungen der Interviewten statt. Von den sechs interviewten pädagogischen Fachkräften waren fünf männlich und eine weiblich.

\subsubsection{Datenfixierung und Aufbereitung}

Die Interviews wurden mit einem digitalen Diktiergerät aufgezeichnet. Die Audioaufnahme wurden je ergänzt durch ein Postskript, in dem die kontextuellen Gegebenheiten des jeweiligen Interviews sowie subjektive Eindrücke und Erinnerungen der Interviewerin festgehalten wurden (zur Bedeutung dieser Daten vgl. Witzel 2000 sowie Breuer et al. 2010, 68 und 133).

Sowohl die Audio- als auch die handschriftlichen Daten wurden in einem nächsten Schritt aufbereitet und als Arbeitsgrundlage verfügbar gemacht, indem sie mit der Transkriptionssoftware f5 transkribiert wurden. Um nicht Gefahr zu laufen, wichtige Daten zu übergehen, wurden Interviews immer komplett transkribiert (vgl. Strauss und Corbin 1996, 14). Um erinnerte nonverbale Geschehnisse während des Interviewverlaufs als Kommentare einfügen zu können, wurden alle Transkripte zeitnah binnen vier Tagen angefertigt.

Für die Transkriptionen wurde eine tabellarische Übersicht von relevanten Transkriptionsregeln erstellt und im Verlaufe der ersten Transkription an die Erfordernisse angepasst. Die verwendeten Transkriptionsregeln sind angelehnt an das von Dresing und Pehl $(2013,21 \mathrm{f} \text {. })^{12}$ vorgeschlagene einfache Transkriptionssystem, bei dem ein schneller Zugang zum Gesprächsinhalt im Vordergrund steht und demzufolge bewusst auf übermässige Details verzichtet wird (vgl. Flick 2002, 253). Da die Aufzeichnung und Transkription eines üblicherweise flüchtigen Gesprächs bereits zu ersten Interpretationen und damit einer «anderen Version des Geschehens» (Flick 2002, 255) führt, wurde im Transkriptionsprozess auf eine entsprechende Sensibilität für Gesprächsatmosphäre, Gesprächsdynamik und Personencharakteristik geachtet (Breuer et al. 2010, 68). Für eine qualitativ hochwertige Transkription wurden die Audioaufnahmen ausserdem mehrfach unter bestimmten Gesichtspunkten (Verständnis, Pausendauer, Dialektglättung etc.) zur Kontrolle gegengehört sowie abschliessend pseudonymisiert (Flick 2002, 253f.).

Das im Rahmen des Forschungsprojekts auszuwertende Datenmaterial umfasste zehn Transkripte und Postskripte der episodischen Interviews mit zwölf pädagogischen Fachkräften (2012/13) und später weitere sechs Transkripte aus Interviews mit sechs pädagogischen Fachkräften (2017).

12 Zur Beurteilung der Qualität des Transkriptionssystems von Dresing/Pehl siehe die vier Gütekriterien (1) Praktikabilität, (2) Ausbaufähigkeit und Flexibilität, (3) Erlernbarkeit und (4) Lesbarkeit von Przyborski und Wohlrab-Sahr $(2010,164)$ und vgl. Flick $(2002,253)$. 


\subsubsection{Computerunterstützte Analyse}

Im Datenanalyseprozess - auf den ich im Anschluss eingehen werde - habe ich mit der Software MAXQDA gearbeitet. Die Software eignet sich laut Kuckartz besonders für das theoretische Kodieren, «denn häufig ist es gerade die Grounded Theory, die bei den Entwicklern der Programme als Leitbild im Hintergrund präsent war, und insofern ist die Software genau für diesen Typ der Analyse konzipiert» (Kuckartz 2010, 82). Assmann macht dies zum Beispiel daran fest, dass es eine Funktion für In-vivoKodes gibt (Assmann 2013, 216), ein für das theoretische Kodieren wichtiges Werkzeug, das es ermöglicht, den natürlichen Sprachgebrauch der interviewten Person als Kode festzuhalten (vgl. Knoll-Krist 1985). Auch aus Sicht der Forschungsdisziplin findet sich ein Argument für die Nutzung von MAXQDA: Laut Kuckartz zählen die Erziehungswissenschaft, die Sozialarbeit und Sozialpädagogik sowie die Bildungsforschung mit ihren Fragen nach subjektiven Bedeutungen zu den Hauptanwendungsfeldern von QDA-Software (Kuckartz 2010, 16). In diesem Zusammenhang eigne sich eine Software besonders für die Auswertung verschiedener Datenformen, wie sie in den oben genannten Disziplinen erhoben werden (Kuckartz 2010, 17).

Der Einwand, dass der Einsatz von Software bisweilen zu «technischen Verkürzungen» führe, die die Anwendung «zum Teil in die Nähe inhaltsanalytischer Verfahren bringen», sei eher auf die Ungeübtheit im Umgang mit Kodierprozess und Software zurückzuführen (Przyborski und Wohlrab-Sahr 2010, 185). Dem Argument der Automatisierung von Kodierprozessen widerspricht Kuckartz: Zwar ermögliche MAXQDA eine Stichwortsuche und auch einfache Vergleiche seien möglich, lediglich komme die Software hier schnell an ihre Grenzen, so dass die Denkarbeit, also die

«intellektuelle Kodierung», immer noch die Forscherin leisten müsse (Kuckartz 2010, 57). Eine Computerunterstützung steigert folglich vor allem die Effizienz, da bestimmte Prozesse im Kodierungs- und Kategorisierungsprozess maschinell berechnet werden und Daten somit schnell zugänglich sind (vgl. Kuckartz 2010, 19). Zum anderen «versprechen die QDA-Programme einen erheblichen Zugewinn an Qualität: Kategoriensysteme, Memos und codierte Textsegmente sind leicht zugänglich und machen es möglich, die Resultate qualitativer Forschung nachzuprüfen» (Kuckartz 2010, 20).

Die Summe dieser Merkmale legte die Verwendung der Software MAXQDA für die Auswertung der vorliegenden Daten nahe. Insbesondere für zwei Prozessschritte wurde MAXQDA in der vorliegenden Arbeit verwendet. Dies waren der gesamte Kodier- und Kategorisierungsprozess sowie für das Verfassen von Memos:

Textpassagen wurden mit Hilfe des Programms markiert, codiert und später systematisiert, indem sie zum Beispiel zu Kategorien zusammengefasst, hierarchisiert, vernetzt, umbenannt oder ausdifferenziert wurden. Bei der Suche nach Überschneidungen von Kategorien oder nach komplexen Mustern von Kategorien wurde 
unter anderem mit den visuellen Darstellungsmöglichkeiten gearbeitet. Ausserdem hilfreich waren die lexikalische Suche nach Worten und Wortkombinationen in den Texten sowie der Einsatz von Variablen als Selektionskriterien und das Textretrieval. Dabei machte ich mir regelmässig bewusst, dass MAXQDA - wie alle Analyseprogramme - eine bestimmte Darstellungsstruktur haben, die die Denkleistung kanalisiert (Flick 2002, 377). Neu emergierende Ideen und Gedanken habe ich deshalb stets hinsichtlich der den Daten immanenten logischen Struktur und teilweise mit analogen Methoden überprüft ${ }^{13}$.

Besonders stark nutzte ich die Möglichkeit, Ideen, Hypothesen und Theorien in Form von Memos festzuhalten. Hierzu habe ich mir ein eigenes Memo-System entwickelt $^{14}$ und den jeweiligen Memoarten die vorgegebenen Icons zugeordnet. In Form von orangenen Dokumentmemos wurden beispielsweise die zu den jeweiligen Transkripten gehörigen Postprotokolle und Notizen zum Interviewverlauf festgehalten. So konnte während der Datenanalyse schnell auf diese zurückgegriffen werden. In blauen Codememos wurden Codes definiert und Ankerbeispiele festgehalten sowie Auffälligkeiten, zum Beispiel mögliche Zusammenhänge und Widersprüche, notiert. Mit «T» waren freie, das heisst unverlinkte Theoriememos markiert. Theoriememos stellten Überlegungen zu theoretischen Verknüpfungen, zu den sogenannten sensibilisierenden Konzepten dar. Das Verwaltungssystem von MAXQDA ermöglichte einen schnellen Zugriff auf diese Memos sowie deren Systematisierung und erleichterte mir insofern die Anfertigung dieser Arbeit.

\subsubsection{Anwendung der Schritte des theoretischen Kodierens}

Im Folgenden soll die Umsetzung der Kodierschritte von offenem und axialem Kodieren vorgestellt werden. Weder die beiden Kodierschritte noch die verwendeten Techniken des Fragenstellens, Vergleichens und Dimensionalisierens waren aus den bereits genannten Gründen in der Praxis eindeutig zu trennen.

Um im Rahmen des offenen Kodierens das Material aufzubrechen, habe ich verschiedene Techniken angewandt. Bei der Analyse der Interviewdaten bin ich zunächst sehr feinschrittig vorgegangen (später dann etwas gröber). Zuerst gliederte ich einen Satz in logisch erscheinende Kontexteinheiten und interpretierte diese dann einzeln. Die Interpretationen wurden in Form von Memos festgehalten und um erste theoretische Überlegungen ergänzt. Beispielsweise erschien es mir zu einem Zeitpunkt wichtig, die Arbeit von Jugendarbeiterinnen und Jugendarbeiter mit der Arbeit von Handwerkerinnen und Handwerkern zu vergleichen, um mir unterschiedliche Werkzeuge,

13 Eine Übersicht zu Legitimation und Grenzen von MAXQDA in der akademischen Forschung gibt Schmieder 2009.

14 Ich nahm zu mehreren Kolleginnen, die Erfahrung in der Arbeit mit MAXQDA hatten, Kontakt auf, um von ihnen zu erfahren, wie sie die verschiedenen Memofunktionen im Kodierprozess effektiv nutzten. Dabei zeigte sich, dass viele die gebotenen Memo-Möglichkeiten nicht oder nur rudimentär nutzten. 
Methoden, Schritte im Arbeitsprozess etc. zu verdeutlichen. An späterer Stelle verfolgte ich daran anknüpfend den Begriff der Methode, indem ich verschiedene Definitionen und Abgrenzungen des Begriffs recherchierte. Diese Begriffsklärung führte letztlich dazu, dass ich den Begriff der Methode durch den Begriff der Methodik ersetzte. Anschliessend wies ich einzelnen gröberen Textabschnitten Kodes zu. Diese entstammten teilweise, um mit Kelle und Kluge zu sprechen, «empirisch nicht gehaltvollen abstrakten theoretischen Konzepten», wurden aber ebenso auch in vivo generiert (Kelle und Kluge 2010). Dieses Vorgehen wandte ich zunächst jeweils fallintern an, um die Kodes in einem folgenden Schritt dann fallübergreifend zu suchen, zu vergleichen und teilweise neu zu clustern oder umzubenennen. Hierbei ergaben sich bereits erste Kategorien und mögliche Dimensionen. Zugleich erwiesen sich bei diesen fallübergreifenden Analysen viele Kodes als nicht geeignet. Einige erforderten eine Differenzierung, während viele sich als zu spezifisch herausstellten und abstrakter formuliert werden mussten, um fallübergreifend gültig sein zu können. Zur Unterstützung nutzte ich in diesem Prozessschritt besonders stark die Memo-Funktion für Codememos.

Parallel zu diesen Schritten im Kodierprozess führte ich je Interview ein Memo, in dem ich Interpretationen zu Eigenheiten der Interviewperson oder des Interviewverlaufs festhielt. Eine Auffälligkeit, die beispielsweise festgehalten wurde, war das Wechseln zwischen Du und Sie bei einem Interviewpartner. Die Trennung in eine analytische Ebene des Sinns und der Form diente mir vor allem der späteren Überprüfung, Hinterfragung oder Untermauerung einzelner Argumente.

Der Umgang mit den unterschiedlichen Datensorten, die durch die episodischen Interviews generiert wurden, fiel mir vergleichsweise leicht. Definitionen und kognitivsemantisches Wissen liessen sich sehr gut vom episodisch-narrativen Anteilen in der Interviews trennen und vergleichen. Inhaltlich führte dies beispielsweise zu der überraschenden ersten Erkenntnis, dass das Handeln mit Social Media und auf SMP - wenn überhaupt - nur in geringem Mass als pädagogisch wahrgenommen wird, während zugleich alle Narrationen pädagogisches Handeln beschreiben.

Mit zunehmender Übung bin ich für die erste Interpretation und Zuweisung von Kodes tendenziell absatzweise vorgegangen und habe lediglich an bedeutsam erscheinenden Stellen eine Feinanalyse von einzelnen Sätzen vorgenommen. Des Weiteren habe ich, um das umfangreiche Datenmaterial zu bewältigen, auch auf technische Funktionen wie die lexikalische Suche, zum Beispiel nach dem Begriff «Beziehung) oder (Vertrauen), sowie die Funktion der Autokodierung verwendet. Letztere hob ich farblich von den bisherigen Kodes ab und nahm selbstverständlich auch eine manuelle Überprüfung vor. Erneut analysierte und interpretierte ich zunächst fallintern und später fallübergreifend.

Das Kategoriensystem folgte zu diesem Zeitpunkt noch überwiegend der Logik des Interviews bzw. des Interviewleitfadens. Um letztlich ein Kategoriensystem 
unabhängig von der Interviewlogik zu entwickeln und um die Zusammenhänge verschiedener Kodes, Dimensionen und Kategorien bildlich und übersichtlich darzustellen, erstellte ich schliesslich sowohl bei der fallinternen wie der fallübergreifenden Analyse im Zusammenhang mit den empfohlenen heuristischen Instrumenten des Fragenstellens und des Dimensionalisierens handschriftliche Mindmaps und Tabellen. Dadurch ergab sich bereits die Bestätigung einiger Hypothesen und Klärung offener Fragen, die sich en passant im Forschungsprozess auftraten. Beispielsweise zeigte sich deutlich, dass sich die Jugendarbeiterinnen und Jugendarbeiter bei der Wahl der SMP uneingeschränkt an der Nutzungspräferenz der jeweiligen jungen Menschen orientieren.

Des Weiteren eruierte ich mit Hilfe des paradigmatischen Modells (Strauss und Corbin 1996, 75) den Gehalt verschiedener emergierter potenzieller Phänomene. Beispielsweise wurde das Kodierparadigma genutzt, um den «Gehalt» von Phänomen zu bestimmen.

Aufgrund der durch die oben genannten Prozedere entstandenen überwältigenden Datenmenge fiel es mir schwer, darauf zu vertrauen, dass ich aus der Fülle der verschiedenen Datensorten (Kodes, Dimensionen und Kategorien sowie Ideen, Konzepte und Memos) ein gewichtiges Phänomen identifizieren könnte. Kennzeichnend für den Einsatz des paradigmatischen Modells im Kontext der vorliegenden Forschungsarbeit und zugleich grösster Gewinn bei der Bewältigung der Menge an emergierenden Phänomenen war, dass diese Visualisierung stets sehr gut veranschaulichte, dass ein vermeintliches Phänomen empirisch nicht gehaltvoll genug und somit zu verwerfen war.

\subsubsection{Reflexion}

Wie bereits dargelegt wurde, empfiehlt Breuer (2010) die Methodik der reflexiven Grounded Theory insbesondere bei Forschungsprojekten, die «Züge persönlicher Projekte» haben und bei denen die Forschenden ein gewisses Mass an «identifikatorischem Herzblut» mitbringen (Breuer et al. 2010, 10). Die vorliegende Arbeit ist deshalb auch ein Spiegel des eigenen Entwicklungsprozesses, den es ebenso wie das Vorgehen im Forschungsprozess iterativ zu reflektieren galt. Breuer empfiehlt, neben der Beantwortung der von ihm formulierten selbstreflexiven Fragen, verschiedene Methoden, um die personale und soziale Selbst-/Reflexivität anzuregen:

Forschungstagebuch: Ein Forschungstagebuch ist Breuer zufolge ein Werkzeug, das die Forschenden «begleitend zum Arbeitsprozess dazu anhält, in systematischer Weise die Transaktionen zwischen Person, Thema, Forschungspartnern und -feld, Kontext etc. aufmerksam zu registrieren, in Worte zu fassen, schriftlich festzuhalten und selbst-/reflexiv zu analysieren» (Breuer et al. 2010, 129). Des Weiteren könne es auch als dokumentarische Stütze der Entwicklung des Forschungsprozesses dienen. 
Dementsprechend sollten alle für die Forschungsarbeit potenziell einschlägigen Gedanken, Assoziationen, Erlebnisse, Gefühlsregungen, Erfahrungen und Probleme darin notiert werden. Breuer schlägt auch vor, darin Memos, Präkonzepte und Postskripte festzuhalten (Breuer et al. 2010, 130).

Im Rahmen der vorliegenden Forschungsarbeit habe ich ein bzw. mehrere Forschungstagebücher geführt. Darin wurden entgegen Breuers Empfehlung jedoch keine Memos oder Postskripte festgehalten, da diese an geeigneterer Stelle in MAXQDA abgespeichert wurden. Dies gilt ebenso für die sensibilisierenden Konzepte, die in Form von Exzerpten im Literaturverwaltungsprogramm JabRef abgespeichert wurden.

Die Forschungsnotizen enthielten vielmehr Gedanken zur Entwicklung der Forschungsfrage und methodischem Design und Umsetzung der Forschungsmethodik. Später verschob sich dies und ich notierte vor allem Feedbacks von Diskussionsvorträgen, Gedanken zu möglichen Kategorien und ihren Zusammenhängen oder Phänomenen. Durchgängig enthalten die Forschungstagebücher Literaturhinweise sowie Notizen, die sich mit der Lösung von forschungspraktischen und -technischen Problemen beschäftigen, zu Beginn waren dies beispielsweise die eigene Rollendefinition, später die Entwicklung eines praktikablen Memosystems oder die Handhabung und bestimmte Einstellungen der verwendeten Textverarbeitungssoftwares Latex und Lyx.

Die Forschungstagebücher wurden im Laufe des langwierigen Forschungsprozesses, in dem die vorliegende Arbeit verfasst wurde, und insbesondere nach längeren Pausen genutzt, um sich wieder «einzudenken». Sie wurden aber auch genutzt, um einzelne theoretische Überlegungen, die in bestimmten Phasen des Forschungsprozesses angestellt wurden, nochmal zu überprüfen oder zunächst verworfene Ideen doch aufzugreifen. Die Forschungstagebücher unterlagen somit auch einem ständigen Re-Writing-Prozess: Gedanken wurden abgeschrieben, neugeschrieben oder weitergeschrieben.

Kollegialer Austausch: Breuer betont mehrfach, wie wichtig es sei und welch positiv- bereichernde Erfahrungen Kolloquien oder Arbeitswerkstätten brächten. Dies gelte, «auch wenn die inhaltlichen Themen der Teilnehmer heterogen und ihr Bearbeitungsstand unterschiedlich fortgeschritten» seien (Breuer et al. 2010, 134).

Im Rahmen der vorliegenden Forschungsarbeit habe ich von dieser Art, meine Forschungserkenntnisse zu reflektieren, am meisten profitiert. Die Bedeutung, die ich dem kollegialen Austausch zuschreibe, bildet sich auch in der blossen Anzahl an Forschungskolloquien und Interpretationsgruppen ab, in denen ich im Verlauf meines langwierigen Forschungsprozesses partizipierte. Darauf, sie alle hier zu benennen, soll verzichtet werden, jedoch möchte ich exemplarisch die Arbeitsweise des von mir selbst organisierten interdisziplinären Forschungsteams «FoTe» kurz darstellen. 
Die Gruppe wurde im Jahr 2013 gegründet, nachdem ich über die Mailingliste des «Forum Qualitative Sozialforschung» nach ebenfalls qualitativ forschenden Interessierten aus dem Berliner Raum gesucht hatte. Sie bestand aus fünf Forschenden mit soziologischen, kommunikations- oder medienwissenschaftlichen Verankerungen. Die Treffen fanden regelmässig einmal monatlich statt und dauerten ca. zwei Stunden, in deren Verlauf stets zwei Projekte bearbeitet wurden. Zur Vorbereitung auf diese wurde das entsprechende Material fünf Tage im Voraus an die Gruppenmitglieder versandt. Im Fokus der Treffen stand meist die Interpretation von bestimmten Textsegmenten, der Vergleich dieser mit anderen Textsegmenten oder die Kategorisierung von Erkenntnissen.

Diese von Breuer genannten Methoden dienten während des gesamten Forschungsprozesses dem «Zurücktreten» und der Einnahme eines distanzierten und reflektierten Standpunktes auf entweder die aktuelle Forschungssituation und aktuelle Erkenntnisse oder auf den gesamten Forschungsprozess.

\subsubsection{Gütekriterien und Kontrolle}

Für Strauss und Corbin ist die Nachvollziehbarkeit des Forschungsverfahrens von hoher Priorität: «Jede Art von Entdeckung entwickelt ihren eigenen Standard - und eigene Verfahren, um diesen Standard zu erreichen. [...] Wichtig ist nur, alle diese Kriterien explizit zu machen» (Strauss und Corbin 1996, 215). Um Lesenden der vorliegenden Forschungsarbeit zu ermöglichen, die im Forschungsprozess getroffenen Entscheidungen und Analysen nachzuvollziehen (vgl. Strauss und Corbin 1996, 217), wurde in den vorherigen Abschnitten versucht, in angemessenem Masse über die Umsetzung der Forschungsmethoden Auskunft zu geben.

Darüber hinaus orientierte ich mich in der Arbeit an den von Steinke entwickelten sogenannten Kernkriterien (Steinke 2013,323), auf die auch Breuer hinsichtlich der Bewertung der Qualität von Untersuchungen nach der (Reflexiven) Grounded Theory-Methodik verweist (Breuer et al. 2010, 109):

1. Der von Strauss und Corbin betonte Aspekt der (intersubjektiven) Nachvollziehbarkeit steht auch in Steinkes Kriterienkatalog an erster Stelle. Sie formuliert diesen Punkt noch aus und fordert eine Dokumentation des Forschungsprozesses bezüglich Vorverständnis, Erhebungsmethoden und -kontext, Transkriptionsregeln, der Daten, der Auswertungsmethoden, der Informationsquellen, von Entscheidungen und Problemen und der Kriterien, denen die Arbeit genügen soll. Dem wurde mit dem Kapitel 2 "Umsetzung der Methoden in der Forschungspraxis» entsprochen. Auch die Anwendung kodifizierter Verfahren - wie sie in der vorliegenden Forschungsarbeit verwendet wurden - sowie die Interpretation in Gruppen gehören für Steinke zur Herstellung einer gewissen Intersubjektivität. Mit der Methode des sogenannten peer debriefing, bei dem ich im Rahmen einer bzw. 
mehrerer (interdisziplinärer) Kodier-/Interpretationsgruppen meine Daten analysierte, werde ich diesen Kriterien gerecht.

2. An zweiter Stelle im Kriterienkatalog benennt Steinke die Indikation des Forschungsprozesses. Die Indikation, also die Angemessenheit, umfasst das qualitative Vorgehen, die Methodenwahl, die Wahl der Transkriptionsregeln und der Samplingstrategie, methodische Einzelentscheidungen sowie die Bewertungskriterien.

3. Ein weiteres Kriterium ist die empirische Verankerung: Der Forschungsprozess sollte laut Steinke so angelegt sein, «dass die Möglichkeit besteht, Neues zu entdecken und theoretische Vorannahmen des Forschers in Frage zu stellen bzw. zu modifizieren» (Steinke 2013, 328). Dass diesem Kriterium Genüge getan wurde, zeigt sich in der vorliegenden Arbeit an verschiedenen Stellen, beispielsweise wurde zunächst nur ein Forschungsinteresse bezüglich des professionellen Handelns von pädagogischen Fachkräften der OKJA im Kontext Social Media formuliert und die Forschungsfrage erst schrittweise und relativ spät im Forschungsprozess konkretisiert. Dementsprechend wurden auch die Erhebungsmethoden nicht im vorhinein festgelegt, sondern im Verlauf des Forschungsprozesses ausgewählt.

4. Die Limitation benennt Steinke als viertes Kriterium. Gemeint ist der Einsatz von Methoden der Fallkontrastierung, um die Grenzen des Geltungsbereichs der Ergebnisse abzustecken. Diesem Punkt wurde in der vorliegenden Arbeit nur bedingt entsprochen, denn es war von Beginn an festgelegt, sich auf die Trias: professionelles Handeln pädagogischer Fachkräfte - OKJA - Social Media zu fokussieren. Weitere Limitationen hinsichtlich der Generalisierbarkeit der Ergebnisse ergeben sich möglicherweise aus der lokalen Nähe (Berlin und Berliner Umland) und den von den pädagogischen Fachkräften genutzten Plattformen (Facebook und WhatsApp). Im Fazit und Ausblick wird dennoch der Blick geöffnet werden und der Versuch unternommen, auf theoretischer Basis und unter Berücksichtigung von weiteren Perspektiven Aussagen zum Geltungsbereich bzw. zur Übertragbarkeit der Erkenntnisse zu treffen.

5. An fünfter Stelle im Kriterienkatalog benennt Steinke die Kohärenz, das bedeutet eine in sich stimmige, generierte Theorie und einen offenen Umgang mit Widersprüchen und offenen Fragen.

6. Der vorletzte Punkt in Steinkes Kriterienkatalog ist die Relevanz, also der pragmatische Nutzen, den die Ergebnisse für Wissenschaft und/oder Praxis mit sich bringen. Dieser Nutzen war im Rahmen der vorliegenden Forschungsarbeit immer leitend. Ich versuchte in meinen Publikationen stets den Spagat, die produzierten Erkenntnisse sowohl für Wissenschaftlerinnen und Wissenschaftler als auch für Praktikerinnen und Praktiker gewinnbringend aufzubereiten. Entsprechendes Feedback durch die Herausgebenden oder Reviewer bestätigte mich darin, dass mir dies gelungen ist. 
7. Der Kriterienkatalog schliesst mit dem Punkt der reflektierten Subjektivität und umfasst laut Steinke folgende zu reflektierende Unterpunkte: Vertrauensbeziehungen zu Informantinnen und Informanten, Selbstbeobachtung, persönliche Voraussetzungen und Reflexionen während des Feldeinstiegs. Dem Kriterium der Reflexion ist in den obigen Ausführungen in allen Unterpunkten Geltung getragen worden und bedarf an dieser Stelle keiner weiteren Ausführungen.

Welche Phänomene der Nutzung von SMP durch pädagogische Fachkräfte in der OKJA mit Hilfe der vorigen dargelegten, empirischen Methoden identifiziert werden konnten, zeigen die folgenden Publikationen. Die Ergebnisse, die sukzessive erarbeitet wurden, werden zusätzlich in «Forschungsergebnisse und Schlussfolgerungen» in einer Querschnittsbetrachtung als zentrale Forschungsergebnisse geordnet.

\section{Zusammenfassung der zentralen Forschungsergebnisse}

Im Fokus des vorliegenden Forschungsprojekts stand das Handeln von pädagogischen Fachkräften der Offene Kinder- und Jugendarbeit (kurz OKJA) im Hinblick auf das Phänomen Social-Media-Plattformen (kurz SMP). Das zunächst offen formulierte Forschungsinteresse, SMP als Instrumente pädagogischen Handelns in der OKJA untersuchen zu wollen, konkretisierte sich im Verlauf des Forschungsprozesses sukzessive hinsichtlich eines Fokus auf professionelles pädagogisches Handeln in der Nutzung von SMP. Diese Entwicklung erfolgte jeweils basierend auf den gewonnenen Erkenntnissen, wie in «Social Media in der Offenen Kinder- und Jugendabreit - Manteltext Einleitung» bereits dargelegt wurde.

Hier werden nun die zentralen Ergebnisse des vorliegenden Forschungsprojekts resümierend zusammengefasst, anschliessend werden daraus Konsequenzen für das professionelle Handeln in der Praxis und Forschungsdesiderate für die weitere wissenschaftliche Auseinandersetzung abgeleitet. Die Einzelergebnisse lassen sich den folgenden drei Themenbereichen zuordnen.

\subsection{Strukturelle Bedingungen der OKJA}

Die Nutzung von SMP durch pädagogische Fachkräfte der OKJA wird von jungen Menschen akzeptiert. Bei entsprechender Zurückhaltung und sensibler Nutzung der erhaltenen Informationen hat die Nutzung grosses pädagogisches Potenzial. Die OKJA stellt mit ihren Strukturmerkmalen einen besonderen Rahmen für eine pädagogische Nutzung von SMP dar, wobei das Strukturmerkmal der Diskursivität, besonders bedeutsam ist, denn gemeinsam ausgehandelte Regeln zum Umgang mit SMP sind die Basis für ein akzeptierendes Miteinander. Die Strukturbedingungen der OKJA bieten pädagogischen Fachkräften zudem in besonderem Masse die Möglichkeit, 
vertrauensvolle Arbeitsbeziehungen aufzubauen. Sie können insofern eine potenziell bedeutsame Rolle als Vorbilder für junge Menschen im Hinblick auf das Medienhandeln einnehmen. Als Vorbilder können sie die jungen Menschen in ihrem Medienhandeln begleiten, ihnen gegebenenfalls helfen dieses zu korrigieren und sie zum Reflektieren anregen.

\subsection{Herausforderungen für die Professionalisierung}

Pädagogische Fachkräfte selbst nutzen SMP intuitiv im Sinne eines Learning by Doing und orientieren sich dabei zunächst an ihrer privaten Nutzung. Dies zeigt sich in Wissensdefiziten zum Beispiel bezüglich der Funktionsoptionen, die Platt- formen bieten, in mangelnder professioneller pädagogischer Einordnung ihres Handelns, aber auch in einem gering ausgeprägten Bewusstsein über die Konsequenzen, die ihr Handeln für die Arbeitsbeziehungen zu den jungen Menschen oder die eigene professionelle Distanz haben kann. Zu kaum revidierbaren Grenzaufweichungen und persönlichen Verflechtungen kommt es auch unter anderem durch die Nutzung privater Mobilgeräte. Es kann festgehalten werden, dass sich die Handlungsfacetten einer pädagogischen SMP-Nutzung im Verlauf schleichend ausdehnen, die Nutzung mehr Zeit in Anspruch nimmt als geplant und es vielfach zu nichtantizipierten Nebeneffekten kommt. Die sich aus der Nutzung von SMP ergebenden ethischen und datenschutzrechtlichen Schwierigkeiten werden als besonders zu reflektierende Herausforderungen für die pädagogischen Fachkräfte identifiziert. Im Hinblick auf den Professionalisierungsdiskurs zur Nutzung von SMP durch pädagogische Fachkräfte ist es von zentraler Bedeutung, sich mit ihnen auseinander zu setzen. Insgesamt erfordert die Nutzung von SMP eine stetige und reflektierte Anpassung der pädagogischen Handlungsstrategien auf Seiten der pädagogischen Fachkräfte. Auch die Tatsache, dass sich die jungen Menschen ständig neuen SMP zuwenden, erfordert die Entwicklung generalisierbarer und somit auf neue Social-Media-Phänomene anwendbare Handlungsstrategien der pädagogischen Fachkräfte. Um das Handeln diesbezüglich zu entindividualisieren und entlasten, also zu professionalisieren, benötigen die pädagogischen Fachkräfte Unterstützung durch ihre Träger oder entsprechende Fachverbände.

\subsection{Pädagogisches Handeln in pädagogischen Räumen}

Eine sich in der Forschungsliteratur und Praxisberichten abzeichnende Dysbalance zwischen sozial-interaktiven und informativ-selbstdarstellenden Tätigkeiten - zugunsten letzterer - stellt sich in der empirischen Analyse tendenziell als «begrifflicher Mangel» heraus. Es wird vermutet, dass auf Grund fehlender pädagogischer Begrifflichkeiten, um das pädagogische Handeln zu beschreiben, auf bekannte Begriffe 
aus dem Marketing (Kundenbindung - Besucherbindung) zurückgegriffen wird. In der Analyse zeigt sich ein grosser, jedoch zunächst implizit bleibender, pädagogischer Gehalt in der SMP-Nutzung. Beim Aufbau von Arbeitsbeziehungen zwischen pädagogischen Fachkräften und jungen Menschen oder der Herstellung eines Zugehörigkeitsgefühls hat die Nutzung von SMP beispielsweise pädagogische Bedeutung. Bei dem Versuch, SMP als Instrumente pädagogischen Handelns zu kategorisieren, stellte sich heraus, dass diese Einordnung zu kurz greift und es sowohl für den theoretischen Professionsdiskurs als auch die Argumentation und Reflexion in der Praxis förderlicher ist, SMP als pädagogische Handlungsräume zu betrachten.

\section{Konsequenzen für professionelles Handeln}

Die Ergebnisse haben deutlich gezeigt, dass die OKJA mit ihren Strukturbedingungen gute Voraussetzungen bietet, SMP in die pädagogische Arbeit zu integrieren. Jedoch gibt es im professionellen Handeln der Fachkräfte selbst noch Entwicklungsbedarfe, denn auf Grund eines intuitiven und teilweise weitgehend unreflektierten Herangehens werden Potenziale nicht ausgeschöpft, rechtliche und ethische Grenzen überschritten.

Um zukünftig die Nutzung von SMP durch pädagogische Fachkräfte der OKJA zu professionalisieren, bedarf es daher eines verstärkten Reflexionsbedarfs des eigenen pädagogischen Handelns sowie der Schaffung von angemessen professionellen Rahmenbedingungen.

\subsection{Reflexion}

Durch die Nutzung von SMP in der pädagogischen Arbeit lassen sich neue Reflexionsbedarfe für pädagogische Fachkräfte ableiten. Strenggenommen sind es keine neuen Reflexionsbedarfe per se, denn auch früher schon mussten pädagogische Fachkräfte über den fachlichen Einsatz ihrer Methoden und Werkzeuge15 reflektieren. Werden SMP allerdings in den Methoden- und Werkzeugkoffer aufgenommen, eröffnen diese - im wortwörtlichen Sinne - neue Dimensionen von Chancen und Risiken, die in dieser Art und Weise neu sind und die einen besonderen Reflexionsbedarf hervorrufen.

Beispielsweise wird es durch die Nutzung von SMP erforderlich, zwischen der leichteren Erreichbarkeit der jungen Menschen und deren schützenswerten Daten abzuwägen. Im Hinblick auf die Nutzung von SMP und deren Gefahrenpotenzialen müssen die pädagogischen Fachkräfte besonders sensibel und weitsichtig abwägen,

15 Im Sinne der vorherigen Ausführungen bedeutet das nicht, dass SMP als Instrumente gesehen werden sollten. Vielmehr erfordert ja auch die Gestaltung von pädagogischen Räumen ein professionell-reflektiertes Vorgehen. 
ob ihr Einsatz den Interessen und Bedarfen der jungen Menschen und dem gesetzlichen Auftrag entgegenkommt oder entgegensteht.

Sich die Interessen und Bedarfe aller Beteiligten regelmässig zu vergegenwärtigen und situativ abzuwägen, ist die entscheidende - wenngleich nicht neue - Konsequenz, die sich für professionell handelnde, pädagogische Fachkräfte aus der Mediatisierung von Handlungsfeldern der Sozialen Arbeit ergibt. Weder blinde Totalverweigerung gegenüber SMP noch blinder Aktionismus entsprechen einem professionellem Handeln.

Pädagogische Fachkräfte können und müssen situativ über den Einsatz von Methoden und Werkzeugen entscheiden. Ihr situatives Handeln zu reflektieren und das Ergebnis in zukünftiges Handeln einfliessen zu lassen, ist dabei Kennzeichen von Professionalität. Die folgenden exemplarischen Reflexionsfragen ergeben sich aus den Forschungserkenntnissen der vorliegenden Arbeit. Sie stellen ein Hilfsmittel dar, die professionelle Nutzung von SMP zu fördern.

Die Formulierung als Fragen verfolgt den Zweck der Wahrung einer neutralen Position und soll symbolisch dafür stehen, dass die pädagogischen Fachkräfte die finale Entscheidung für oder gegen die Nutzung von SMP im beruflichen Kontext und ggf. deren Ausgestaltung situativ treffen können und müssen.

Die Reflexionsfragen berücksichtigen verschiedene Perspektiven, die jedoch nur aus analytischer Sicht getrennt betrachtet werden sollten. Zuerst sollen die Fragen die Perspektive der jungen Menschen bzw. der Klientinnen und Klienten reflektieren helfen. Da, wie deutlich wurde, auch die Haltung der pädagogischen Fachkräfte eine bedeutende Rolle spielt, werden weitere Reflexionsfragen diese Perspektive beleuchten.

Auf Ebene der Klientinnen und Klienten umfassen die Reflexionsbedarfe sowohl Fragen des Zugangs als auch der Nutzungspraxen und -kompetenzen sowie die damit befriedigten Bedürfnisse; beispielsweise sind dies die folgenden:

\subsubsection{Reflexion der Klientinnen- und Klienten-Perspektive}

- Welche technischen Voraussetzungen liegen bei den Klientinnen und Klienten vor?

- Welche Kompetenzen können bei den Klientinnen und Klienten hinsichtlich der Nutzung vorausgesetzt werden?

- Welche Nutzungspraxen haben die Klientinnen und Klienten?

- Welche Bedürfnisse befriedigen die Klientinnen und Klienten durch die Nutzung von SMP?

- Welche Faktoren schränken ggf. die Nutzung der Klientinnen und Klienten ein?

- Welche alternativen (weniger datenprekären) Möglichkeiten gibt es, die Klientinnen und Klienten zu erreichen? 
- Welche alternativen Möglichkeiten, ihre Bedürfnisse zu befriedigen, kennen die Klientinnen und Klienten?

Die sensibilisierenden Reflexionsfragen hinsichtlich der Perspektive der pädagogischen Fachkräfte umfassen die Antizipation der Einflüsse von SMP auf die Interaktionen und Arbeitsbeziehungen sowie Aspekte der eigenen Haltung gegenüber SMP und eigene Kompetenzen. Exemplarisch sind dies:

\subsubsection{Reflexion der eigenen fachlichen Perspektive}

- Wie beeinflussen SMP den Zugang, die Interaktion und den Dialog zwischen den pädagogischen Fachkräften und den jungen Menschen?

- Wie beeinflussen SMP die Arbeitsbeziehungen zwischen den pädagogischen Fachkräften und jungen Menschen?

- Welche neuen Verantwortungsdimensionen ergeben sich für die pädagogischen Fachkräfte?

- Welche spezifischen Qualifikationen sind nötig, um als pädagogische Fachkraft handlungsfähig zu bleiben?

- Welche fachlichen Standards und Grundhaltungen liegen dem pädagogischen Handeln der Fachkräfte zu Grunde?

- Wie können sich pädagogische Fachkräfte angesichts verschwimmender Grenzen distanzieren?

- Findet kollegialer Austausch auf (kommerziellen) SMP statt?

Indem sich die pädagogischen Fachkräfte beispielsweise mit den vorherigen Reflexionsfragen auseinandersetzen und ihre Reflexionserkenntnisse in Handeln übertragen, führt dies zunehmend zu einer professionell pädagogischen Nutzung von SMP. Da das pädagogische Handeln zu einem entscheidenden Teil aber auch von entsprechend professionellen Rahmenbedingungen beeinflusst ist und sich viele pädagogische Fachkräfte von den Trägern Unterstützung wünschen, folgen nun - als zweite Konsequenz aus den obigen Ergebnissen - Überlegungen, wie diese Rahmenbedingungen aussehen könnten.

\subsection{Schaffung von angemessen professionellen Rahmenbedingungen}

Pädagogische Fachkräfte haben ein Bedürfnis nach Orientierung geäussert. Sie benötigen Leitlinien zur Reduktion von Unsicherheiten und als Entlastung. Da das Handeln von pädagogischen Fachkräften situativen Bedingungen unterliegt, ist es wichtig, dass diese nicht als feste Vorgaben, Regeln oder gar Gesetze in die Autonomie der Fachlichkeit eingreifen. Die pädagogischen Fachkräfte sind die Personen mit der 
grössten Nähe zu der jungen Menschen und brauchen daher auch Freiräume, situativ und den Erfordernissen entsprechend zu entscheiden. Die wenigsten Träger haben bislang entsprechende Leitlinien zur Nutzung von SMP im Allgemeinen. Leitlinien für die pädagogische Arbeit im Speziellen sind mir von Trägerseite gar nicht bekannt. Vielmals findet sich die Nutzung von SMP noch nicht einmal in den EinrichtungsKonzeptionen, so dass bislang nur Absprachen im Team die einzelne pädagogische Fachkraft entlasten. Dies ist deshalb auch als das grösste Defizit zu bezeichnen und lässt einen nachdrücklichen Appell an die Träger formulieren.

Im Folgenden werden erneut - auf Basis der Forschungserkenntnisse der vorliegenden Arbeit - einige exemplarische Reflexionsfragen formuliert. Sie können die Schaffung professioneller Rahmenbedingungen von Seiten der Träger fördern, indem sie deren Interessen, gesetzlich zu erfüllende Aufgaben, organisatorische, fachlichkonzeptionelle und ethische Fragen reflektieren helfen. Unter anderem sind dies:

- Welche Aufgaben und damit zusammenhängende Arbeitsabläufe können mittels SMP vereinfacht werden?

- Welche neuen Verantwortungsdimensionen ergeben sich daraus für den Träger?

- Welche pädagogischen Ansätze der SMP-Nutzung lassen sich im Konzept verankern?

- Wie verändert die Mediatisierung im Allgemeinen und die Nutzung von SMP im Speziellen die pädagogischen Handlungsgrundsätze?

- Kann es durch die Nutzung des Träger-Angebots auf SMP zu (öffentlichen) Stigmatisierungen der Klientinnen und Klienten kommen?

- Von welchem Dienstrechner werden SMP genutzt? Wie wird mit Cookies etc. darauf verfahren?

- Welche Rahmenbedingungen müssen von Trägerseite verändert werden, um den pädagogischen Fachkräften die pädagogische Arbeit zu ermöglichen?

- Gibt es eine Ansprechperson, die die (pädagogischen) Fachkräfte technisch und rechtlich unterstützen und beraten kann?

Die Reflexionsfragen sprechen auch die technische Ausstattung als Rahmen für professionelles Handeln an. Bereits in dem Beitrag «Schleichende Grenzaufweichungen und persönliche Verflechtungen» wurde dazu festgestellt, dass ein WhatsAppBusiness-Account Vorteile für eine professionelle Grenzziehung mit sich bringt, wenngleich die Nutzung von WhatsApp an sich datenschutzrechtliche Konsequenzen und diesbezügliche Fragen aufwirft. Die Anschaffung von Diensthandys bei der Nutzung von MessengerApps kann die pädagogischen Fachkräfte ebenfalls dabei unterstützen, professionell mit Nähe und Distanz umzugehen.

Sowohl für pädagogische Fachkräfte als auch für die Träger sollte die Phase des Learning by Doing und die Zeit des blinden Aktionismus im Sinne von «hauptsache dabei-Sein» vorbei sein. Ebenso sollten sich Skepsis und ein risikoorientierter Blick 
nicht länger in Verschlossenheit niederschlagen, sondern zu einer professionellen Auseinandersetzung mit SMP führen. Dazu sollten Träger und pädagogische Fachkräfte gemeinsam überlegen, wie praktikable Rahmenbedingungen und Leitlinien, die Handlungssicherheit für professionell pädagogisches, autonomes Handeln aussehen könnten und welche weiteren beispielsweise technischen Rahmenbedingungen dafür geschaffen werden müssen.

Ebenso sollten die pädagogischen Fachkräfte und die jungen Menschen im Sinne von Partizipation und Diskursivitätsgrundsatz sich abstimmen und gemeinsame Interaktionsregeln formulieren, bei der die beiderseitigen Grenzen im professionellen Sinne gewahrt bleiben.

Die OKJA mit ihren spezifischen und wertvollen Strukturmerkmalen kann nicht durch Interaktionen auf SMP ersetzt werden und man sollte dies auch nicht versuchen. SMP sollten im Handlungsfeld der OKJA SMP lediglich eine additive Ausweitung bestehender pädagogischer Aktions- oder Handlungsräume darstellen. Ziel der Nutzung von SMP sollte stets sein, die pädagogischen Interaktionen und Arbeitsbeziehungen zu fördern. Dies kann stattfinden,

- indem pädagogische Fachkräfte den jungen Menschen als kompetente Ansprechpartnerinnen und -partner auch zur Verfügung stehen, wenn sich dieser Unterstützungs- bedarf auf Probleme mit SMP bezieht.

- indem organisatorische Aspekte der Arbeit wie Werbung digital oder über SMP erledigt werden, um die gewonnene Zeit dann interaktiv mit den jungen Menschen zu verbringen.

- indem situativ auf Onlineberatung zurückgegriffen wird, wenn ein junger Mensch daran gehindert ist, die JFE persönlich aufzusuchen.

Vor diesem Hintergrund kann und soll die Nutzung von SMP in das professionelle Handeln pädagogischer Fachkräfte integriert werden. Denn nur auf professionelle Weise kann das Potenzial von SMP sinnvoll, angemessen und zum Nutzen aller eingesetzt werden.

\section{Forschungsdesiderate}

Im Fokus der Arbeit stand das professionelle Handeln von pädagogischen Fachkräften der OKJA im Hinblick auf das Phänomen SMP. Die Limitation auf Grund der gewählten Forschungsfoki und -methoden lässt noch viele Forschungsdesiderate unbeantwortet. Beispielsweise fordern die jetzigen Erkenntnisse zu weiteren Forschungen bezüglich der Konsequenzen für die Arbeitsbeziehungen zwischen pädagogischen Fachkräften und jungen Menschen heraus. Kann die Nutzung von SMP zu einer qualitativen Beziehungsintensivierung führen? Darüber hinaus könnte weitere Forschung der viel geäusserten Forderung nachkommen, die Perspektive der jungen 
Menschen auf die pädagogische Nutzung von SMP zu berücksichtigen und gegebenenfalls derjenigen der pädagogischen Fachkräfte gegenüberzustellen. Im Hinblick auf die Professionalität pädagogischer Fachkräfte könnte weitere Forschung die Nutzung von SMP für die eigene berufliche Weiterentwicklung untersuchen. Dies umfasst beispielsweise die Vernetzung via SMP mit Kolleginnen und Kollegen aus dem direkten beruflichen Umfeld, die Recherche auf Fachportalen und in Fachforen oder die Mitgliedschaft in Online(diskussions)gruppen. Wie die Forschungsstandanalyse ergab, fehlte es der pädagogischen Praxis besonders an Wissen um Möglichkeiten und Grenzen. Dieser Aspekt steht in engem Zusammenhang mit der ebenfalls noch zu erforschenden Frage, welche strukturellen Veränderungen die Mediatisierung des pädagogischen Handelns und pädagogischer Handlungsfelder bedingt. Forschungen im Bereich Enterprise 2.0 (Sternberg 2016) zeigen, dass veränderte Kommunikationspraxen Hierarchien innerhalb von Teams und zu Vorgesetzten verändern. Nicht zuletzt stellt sich im Hinblick auf die Professionalität von pädagogischen Fachkräften die Frage, welche medien(-pädagogischen) Kompetenzen pädagogische Fachkräfte (der OKJA) für ihre Arbeit brauchen.

Auf Grundlage der Erfahrungen und Erkenntnisse aus dem vorliegenden Forschungsprojekt böten sich einige Methoden besonders zur Beantwortung einzelner dieser Themenkomplexe an: Zur Erfassung der Konsequenzen für die Arbeitsbeziehungen wäre zu prüfen, ob ein ethnografisches Vorgehen ein Bild über die Interaktionsprozesse im Rahmen der Arbeitsbeziehungen zwischen jungen Menschen und pädagogischen Fachkräften abzugeben vermag. Eine teilnehmende Beobachtung der Interaktionen vor Ort als auch eine Auswertung von Nachrichtenverläufen auf SMP würde ermöglichen die Aktionen und Reaktionen beider Seiten zu erfassen und zu deuten. Ein anschliessendes Interview mit den beteiligten Gesprächspartnerinnen und -partnern könnten dann genutzt werden, um sich über die Deutungen zu verständigen. Das Interview könnte zudem als metadialogisches Werkzeug pädagogischen Wert entfalten und der Beziehungsklärung und -festigung zwischen pädagogischer Fachkraft und jungem Menschen dienen. Zur Erfassung der Perspektive der jungen Menschen auf eine pädagogische Nutzung von SMP böten sich möglicherweise Gruppendiskussionen mit jeweils jungen Menschen und pädagogischen Fachkräften sowie spätere gemischte Gruppen an. Sie könnten nicht nur wissenschaftlich sehr aufschlussreich sein, sondern der Forderung nach diskursiv entwickelten Leitfäden für die gemeinsame Social-Media-Nutzung als Grundstein entsprechen. Die Aktionsforschung könnte sich in günstiger Weise dem Thema der professionellen Weiterentwicklung widmen und gemeinsam mit der Praxis pädagogische Konzepte entwickeln sowie deren Einsatz in der Praxis überprüfen. Zur Frage nach den erforderlichen medien(-pädagogischen) Kompetenzen pädagogischer Fachkräfte müssten zunächst Anforderungen und Zielformulierungen (beispielsweise Demokratiebildung oder Wertevermittlung) von gesetzlicher Seite mit solchen von Trägern und 
pädagogischen Fachkräfte abgeglichen und unter den veränderten Bedingungen der Mediatisierung überprüft werden.

Die vorliegende Arbeit versteht sich auch als Anstoss, diesen weiteren Forschungsthemen nachzugehen, um die Professionalisierung der SMP-Nutzung in der OKJA voranzubringen.

\section{Die Publikationen in der Übersicht}

Die Rahmen des vorliegenden Forschungsprojekts der kumulativen Promotion publizierten schriftlichen Beiträge werden im Folgenden in ihrer chronologischen Reihenfolge aufgelistet:

Stix, Daniela Cornelia. 2014. «Soziale Onlinenetzwerke (k)eine pädagogenfreie Zone?» Deutsche Jugend, Nr. 12: 531-38. https://doi.org/10.3262/DJ1412531.

Stix, Daniela Cornelia. 2015a. «Mehr als Öffentlichkeitsarbeit - außerschulische Jugendarbeit in Sozialen Onlinenetzwerken». Der pädagogische Blick 23 (3): 157-68. https://doi. org/10.3262/PB1503157.

Stix, Daniela Cornelia. 2015b. «Warum Soziale Onlinenetzwerke in der Jugendarbeit noch weiter erforscht werden müssen: Ein Beitrag aus Perspektive der außerschulischen Kinderund Jugendarbeitsforschung». Herausgegeben von Thorsten Junge. Soziale Netzwerke im Diskurs, 213-38. https://doi.org/10.18445/20160127-125534-0.

Stix, Daniela Cornelia. 2018a. «Gute Vorbilder für die Medienkompetenz. Chancen für die Kinder- und Jugendarbeit». Herausgegeben von Katholische LandesarbeitsgemeinschaftKinder- und Jugendschutz NRW e.V. THEMA JUGEND. Zeitschrift für Jugendschutz und Erziehung, Nr. 4: 15-17. http://www.thema-jugend.de/fileadmin/redakteure/THEMA_JUGEND/ TJ_4_2018.pdf.

Stix, Daniela Cornelia. 2018b. «Social Media Platforms as Pedagogical Spaces. The Professional Use of Social Media Platforms in Youth Work». MEDIA EDUCATION - Studi, ricerche, buone pratiche 9 (2): 329-42. https://doi.org/10.14605/MED921809.

Stix, Daniela Cornelia. 2018c. «Soziale Onlinenetzwerke als pädagogische Handlungsräume: Fallbeispiele der Offenen Kinder- und Jugendarbeit zur professionellen Nutzung von Sozialen Onlinenetzwerken». Sozial Extra 42 (3): 12-15. https://doi.org/10.1007/s12054-0180044-0.

Stix, Daniela Cornelia. 2019. «Schleichende Grenzaufweichungen und persönliche Verflechtungen. Strategien im pädagogischen Handeln mit Social-Media-Plattformen in der Offenen Kinder- und Jugendarbeit». Zeitschrift für Sozialpädagogik (ZfSp), Nr. 2: 167-85. https://doi.org/10.3262/ZFSP1902167. 


\section{Literatur}

Alfert, Nicole. 2013. «Facebook als Handlungsraum in der Jugendarbeit - Krise oder strategische Chance für Institutionen?» In Soziale Arbeit in der Krise, herausgegeben von Karin Böllert, Nicole Alfert, und Marc Humme, 85-107. Wiesbaden: Springer VS. https://doi. org/10.1007/978-3-531-19088-4_5.

Alfert, Nicole. 2015. Facebook in der Sozialen Arbeit. Wiesbaden: Springer VS. https://doi. org/10.1007/978-3-658-07100-4.

Aßmann, Sandra. 2013. Medienhandeln zwischen formalen und informellen Kontexten: doing connectivity. Wiesbaden: Springer VS. https://doi.org/10.1007/978-3-658-01940-2.

AWO Bundesverband e.V. 2013. "Social Media Strategiepapier. Leitlinien für die Anwendung von Sozialen Netzwerken für die AWO». https://www.awo-onlineservices.org/index. php?elD=tx_nawsecuredl\&u=0\&file=fileadmin/user_upload/redaktionsservice/Leitlinien_ und_Handbuecher/AWO\%20Bundesverband-Strategiepapier-2013_.pdf\&t=1600851438\&h ash $=$ e63fdd464220f8c46013cc464ce705d20b11bc3f.

Boos, Marc. 2015. «So wollen wir online kommunizieren. 5 Prinzipien für die Online-Kommunikation der Caritas». caritas digital - die Nächsten im Netz (blog). 29. September 2015. https://www.caritas-digital.de/so-will-die-caritas-online-kommunizieren/.

Boos, Marc. 2017. «Netiquette für die Nutzung der Social-Media-Kanäle der Caritas Deutschland». caritas digital - die Nächsten im Netz (blog). 3. August 2017. https://www.caritas-digital.de/netiquette-fuer-die-nutzung-der-social-media-kanaele-der-caritas-deutschland/.

Breuer, Franz, Barbara Dieris, und Antje Lettau. 2010. Reflexive Grounded Theory. Eine Einführung für die Forschungspraxis. 2. Wiesbaden: VS Verlag für Sozialwissenschaften. https:// doi.org/10.1007/978-3-531-92580-6.

Breuer, Franz, Günter Mey, und Katja Mruck. 2011. «Subjektivität und Selbst-/Reflektivität in der Grounded Theory Methodologie». In Grounded Theory Reader, herausgegeben von Günter Mey; Katja Mruck, 2., akt. u. erw., 427-48. Wiesbaden: VS Verlag für Sozialwissenschaften. https://doi.org/10.1007/978-3-531-93318-4_19.

Carstensen, Tanja. 2015. «Neue Anforderungen und Belastungen durch digitale und mobileTechnologien». WSI Mitteilungen, Nr. 3: 187-193.

Charmaz, Kathy. 2011. «Den Standpunkt verändern: Methoden der konstruktivistischen Grounded Theory». In Grounded Theory Reader, herausgegeben von Günter Mey; Katja Mruck, 2., akt. u. erw., 181-205. Wiesbaden: VS Verlag für Sozialwissenschaften. https://doi. org/10.1007/978-3-531-93318-4_9.

Cloos, Peter, Stefan Köngeter, Burkhard Müller, und Werner Thole. 2009. Die Pädagogik der Kinder- und Jugendarbeit. 2., durchges. Wiesbaden: VS Verlag für Sozialwissenschaften. https://doi.org/10.1007/978-3-531-91557-9.

Coyle, Pat. 2011. "Considering The Facebook Effect». 2011. http://www.coylemedia. com/2010/10/26/considering-the-facebook-effect/.

DAK-GESUNDHEIT. 2017. «WhatsApp, Instagram und Co. - so süchtig macht Social Media». https://www.dak.de/dak/download/dak-studie-social-media-nutzung-1968596.pdf. 
Deutscher Caritasverband e.V. 2011. «Social Media Guidelines der Caritas». http://www.caritas. $\mathrm{de} / \mathrm{cms} /$ contents/caritasde/medien/dokumente/zentraledokumente/socialmedialeitlinie/ social_media_leitlinien_caritas_mitarbeiter.pdf.

Deutsches Institut für Vertrauen und Sicherheit im Internet (DIVSI). 2014. «DIVSI U25-Studie. Kinder, Jugendliche und junge Erwachsene in der digitalen Welt». 2014. https://www.divsi. de/wp-content/uploads/2014/02/DIVSI-U25-Studie.pdf.

DOJ - Dachverband offene Jugendarbeit. 2010. «Social Media. Neue Medien und offene Jugendarbeit/Nouveaux médias et animation jeunesse». InfoAnimation 8 (21).

Dresing, Thorsten, und Thorsten Pehl. 2013. Praxisbuch Interview, Transkription \& Analyse. Anleitungen und Regelsysteme für qualitativ Forschende. 5. Eigenverlag.

Ebersbach, Anja, Markus Glaser, und Richard Heigl. 2011. Social Web. 2., völlig überarb. Kons$\operatorname{tanz:}$ UVK.

Ebersbach, Anja, Markus Glaser, und Richard Heigl. 2016. Social Web. 3., überarb. Konstanz/ München: UVK Verl.-Ges.

Flick, Uwe. 2002. Qualitative Sozialforschung. Eine Einführung. 6. Reinbek: rororo.

Flick, Uwe. 2011a. «Das Episodische Interview». In Empirische Forschung und Soziale Arbeit, herausgegeben von Gertrud Oelerich und Hans-Uwe Otto, 273-80. Wiesbaden: VS Verlag für Sozialwissenschaften. https://doi.org/10.1007/978-3-531-92708-4_17.

Flick, Uwe. 2011b. Triangulation. Eine Einführung: eine Einführung. 3., akt. Wiesbaden: VS Verlag für Sozialwissenschaften. https://doi.org/10.1007/978-3-531-92864-7.

Gerodetti, Julia. 2009. «Sozialraum Online Community - Ergebnisse einer Rekonstruktion sozialräumlicher Aspekte von Online Communities am Beispiel www.festzeit.ch». 2009. http:// www.sozialraum.de/sozialraum-online-community.php.

Giesecke, H. 1980. Die Jugendarbeit. 5. völlig neu bearb. München: Juventa.

Giesecke, Hermann. 1997. Die pädagogische Beziehung: pädagogische Professionalität und die Emanzipation des Kindes. Weinheim u.a.: Juventa.

Glaser, Barney G., und Anselm L. Strauss. 1967. The discovery of grounded theory: strategies for qualitative research. Observations. Chicago: Aldine.

Glaser, Barney G., und Anselm L. Strauss. 2006. The discovery of grounded theory. Strategies for Qualitative Research. Reprint. New Brunswick, London: Aldine.

Goffman, Erving. 1959. The Presentation of Self in Everyday Life. Revised. New York: Doubleday.

Kammerl, Rudolf. 2006. «Funktionalität und Dysfunktionalität des Chattens für Beziehungen von 14- bis 16-jährigen Jugendlichen». In MedienPädagogik, 1-22. https://doi. org/10.21240/mpaed/00/2006.03.19.X

Karl, Katherine A., und Joy V. Peluchette. 2011. «Friending Professors, Parents and Bosses: A Facebook Connection Conundrum». Journal of Education for Business 86 (4): 214-22. https://doi.org/10.1080/08832323.2010.507638.

Kelle, Udo, und Susann Kluge. 2010. Vom Einzelfall zum Typus. Fallvergleich und Fallkontrastierung in der qualitativen Sozialforschung. 2. Wiesbaden: VS Verlag für Sozialwissenschaften. https://doi.org/10.1007/978-3-531-92366-6. 
Kleinebrahm, Tobias. 2018. "6 Schritte zu einer lebendigen Facebook-Gruppe». caritas digital - die Nächsten im Netz (blog). 28. Mai 2018. http://www.caritas-digital.de/6-schritte-zueiner-lebendigen-facebook-gruppe/.

Knoll-Krist, D.H. 1985. Profis im Jugendhaus: Identitätsprobleme zwischen Alltagsrealität u. Utopie. Stuttgart: Ed. Cadre.

Korfmacher, Susanne. 2011. «Online-Jugendarbeit. Ergebnisse der Umfrage zur Nutzung der Möglichkeiten von Web 2.0-Tools durch die Berliner Jugendfreizeiteinrichtungen». 2011. https://www.jugendnetz-berlin.de/de-wAssets/docs/04jugendarbeit/Studie_Jugendarbeit_Online_Endfassung.pdf.

Krotz, Friedrich. 2001a. «Die Übernahme öffentlicher und individuelle Kommunikation durch die Privatwirtschaft. Über den Zusammenhang zwischen Mediatisierung und Ökonomisierung». In Medienwirtschaft und Gesellschaft 1, herausgegeben von Matthias Karmasin, Manfred Knoche, und Carsten Winter, 197-217. Münster: LIT.

Krotz, Friedrich. 2001. Die Mediatisierung kommunikativen Handelns: der Wandel von Alltag und sozialen Beziehungen, Kultur und Gesellschaft durch die Medien. Wiesbaden: Westdeutscher Verlag. https://doi.org/10.1007/978-3-322-90411-9.

Krotz, Friedrich. 2006. «Mediatisierung und Globalisierung als Wandlungsprozesse und die so genannte digitale Spaltung. Konzepte und Visionen für eine Medienpädagogik der Zukunft». In Schöne neue Medienwelten, herausgegeben von Ingrid Volkmer und Dieter Wiedemann, 60-69. Bielefeld: GMK.

Krotz, Friedrich. 2007. Mediatisierung. Fallstudien zum Wandel von Kommunikation: Fallstudien zum Wandel von Kommunikation. Wiesbaden: VS Verlag für Sozialwissenschaften. https:// doi.org/10.1007/978-3-531-90414-6.

Krotz, Friedrich, und Iren Schulz. 2013. "Jugendkulturen im Zeitalter der Mediatisierung». In Digitale Jugendkulturen, herausgegeben von Kai-Uwe Hugger, 31-44. Wiesbaden: Springer VS. https://doi.org/10.1007/978-3-531-19070-9_2.

Kruse, Jan. 2011. Reader «Einführung in die Qualitative Interviewforschung». Freiburg.

Kuckartz, Udo. 2010. Einführung in die computergestützte Analyse qualitativer Daten. 3., aktualisierte Aufl. Wiesbaden: VS Verlag für Sozialwissenschaften. https://doi.org/10.1007/9783-531-92126-6.

Kutscher, Nadia, Thomas Ley, und Udo Seelmeyer. 2015. Mediatisierung (in) der Sozialen Arbeit. Baltmannsweiler: Schneider-Verlag Hohengehren.

Löw, Martina. 2001. Raumsoziologie. Suhrkamp. Frankfurt am Main: Suhrkamp.

Lüdtke, Hartmut, und Gustaf Grauer. 1973. Jugend - Freizeit - Offene Tür. Methoden und Daten der empirischen Erhebung in Jugendfreizeitheimen. Weinheim, Basel: Beltz.

Maiwald, Kai-Olaf. 2003. «Stellen Interviews eine geeignete Datenbasis für die Analyse beruflicher Praxis dar?» Sozialer Sinn, Nr. 1: 151-80. https://doi.org/10.1515/sosi-2003-0110.

Mansholt, Malte. 2018. «DSGVO: Warum Sie WhatsApp bald nicht mehr im Job benutzen dürfen». Stern 18. Mai 2018. https://www.stern.de/digital/smartphones/dsgvo-warum-siewhatsapp-bald-nicht-mehr-im-job-benutzen-duerfen-7988916.html. 
McQuail, Denis. 1983. Mass communication theory: an introduction. London u.a.: Sage.

Medienpädagogischer Forschungsverbund Südwest. 2012. Kinder + Medien, Computer + Internet: KIM ...; Basisuntersuchung zum Medienumgang 6- bis 13-Jähriger in Deutschland. Stuttgart. http://www.mpfs.de/fileadmin/KIM-pdf12/KIM_2012.pdf.

Medienpädagogischer Forschungsverbund Südwest. 2013. JIM - Jugend, Information, (Multi-) Media: JIM; Basisuntersuchung zum Medienumgang 12- bis 19-Jähriger. Stuttgart. http:// www.mpfs.de/fileadmin/JIM-pdf13/JIMStudie2013.pdf.

Medienpädagogischer Forschungsverbund Südwest. 2018. JIM - Jugend, Information, (Multi-) Media: JIM ; Basisuntersuchung zum Medienumgang 12- bis 19-Jähriger. Stuttgart. https:// www.mpfs.de/fileadmin/files/Studien/JIM/2018/Studie/JIM_2018_Gesamt.pdf.

Mey, Günter, und Katja Mruck, Hrsg. 2011. Grounded Theory Reader. 2., akt. u. erw. Wiesbaden: VS Verlag für Sozialwissenschaften. https://doi.org/10.1007/978-3-531-93318-4.

Mishna, Faye, Marion Bogo, Jennifer Root, Jami-Leigh Sawyer, und Mona Khoury-Kassabri. 2012. "It just crept in": The Digital Age and Implications for Social Work Practice». Clinical Social Work Journal 40: 277-286. https://doi.org/10.1007/s10615-012-0383-4.

ÖSTERREICHISCHES ROTES KREUZ. 2010. «Rotkreuz-Social-Media-Policy». https://www.roteskreuz.at/fileadmin/user_upload/PDF/Was_wir_tun/Social-Media-Policy.pdf.

Przyborski, Aglaja, und Monika Wohlrab-Sahr. 2010. Qualitative Sozialforschung: ein Arbeitsbuch. 3., korr. München: Oldenbourg.

Royal Society for Public Health (RSPH). 2017. «Status Of Mind: Social media and young people's mental health and well-being». https://www.rsph.org.uk/uploads/assets/ uploaded/62be270a-a55f-4719-ad668c2ec7a74c2a.pdf.

Schmidt, Jan-Hinrik, und Monika Taddicken. 2017. «Soziale Medien: Funktionen, Praktiken, Formationen». In Handbuch Soziale Medien, herausgegeben von Jan-Hinrik Schmidt und Monika Taddicken, 23-37. Wiesbaden: Springer VS. https://doi.org/10.1007/978-3-65803765-9_2.

Schmieder, Christian. 2009. "Computergestützte qualitative Datenanalyse: Technik der Legitimation - Legitimation der Technik: eine qualitative Studie zur Verwendung von MAXQDA in akademischem Forschen». 2009. https://nbn-resolving.org/urn:nbn:de:bsz:25opus-70823.

Steinke, Ines. 2013. «Gütekriterien qualitativer Forschung: ein Handbuch». In Qualitative Forschung. Ein Handbuch, herausgegeben von Uwe Flick, Ernst von Kardorff, und Ines Steinke, 10., 317-31. Reinbek bei Hamburg: Rowohlt-Taschenbuch-Verlag.

Sternberg, René. 2016. Enterprise 2.0 und interne Unternehmenskommunikation. Berlin/Kassel: B\&S Siebenhaar Verlag.

Stix, Daniela Cornelia. 2013. «Checkliste für verantwortungsvolle Jugendarbeit in den Sozialen Onlinenetzwerken“. Eigendruck VJB - Jugend und Familie gGmbH.

Stix, Daniela Cornelia, und Lisa Harneid. 2019. «Social Media und jugendliches Coming-out. Chancen - Risiken - Unterstützungsmöglichkeiten». In Deutsche Jugend 67(9): 387-395. 
Strauss, Anselm. 1991. Grundlagen qualitativer Sozialforschung: Datenanalyse und Theoriebildung in der empirischen soziologischen Forschung. München: Fink.

Strauss, Anselm, und Juliet Corbin. 1996. Grounded Theory: Grundlagen qualitativer Sozialforschung. Weinheim: Beltz.

Strauss, Anselm, Heiner Legewie, und Barbara Schervier-Legewie. 2011. «Forschung ist harte Arbeit, es ist immer ein Stück Leiden damit verbunden. Deshalb muss es auf der anderen Seite Spaß machen». In Grounded Theory Reader, herausgegeben von Günter Mey und Katja Mruck, 2., akt. u. erw., 69-78. Wiesbaden: VS Verlag für Sozialwissenschaften. https:// doi.org/10.1007/978-3-531-93318-4_3.

Thole, Werner, und Ernst-Uwe Küster-Schapfl. 1997. Sozialpädagogische Profis. Beruflicher Habitus, Wissen und Können von PägagogInnen in der außerschulischen Kinder- und Jugendarbeit. Opladen: Leske+Budrich.

Thole, Werner, Peter Cloos, Stefan Köngeter, und Burkhard Müller. 2011. «Ethnographie der Performativität pädagogischen Handelns. Zu den Möglichkeiten, die Konstitutionsbedingungen sozialpädagogischer Handlungsfelder zu erkunden». In Empirische Forschung und Soziale Arbeit: Ein Studienbuch, herausgegeben von Gertrud Oelerich, und Hans-Uwe Otto, 115-36. Wiesbaden: VS Verlag für Sozialwissenschaften. https://doi.org/10.1007/978-3531-92708-4_7.

Wagner, Ulrike, Niels Brüggen, und Christa Gebel. 2009. «Web 2.0 als Rahmen für Selbstdarstellung und Vernetzung Jugendlicher - Analyse jugendnaher Plattformen und ausgewählter Selbstdarstellungen von 14- bis 20-Jährigen». 2009. https://www.jff.de/fileadmin/user_upload/jff/projekte/konvergenzstudien/Bericht_Web_2.0_Selbstdarstellungen_JFF_2009. pdf.

Wagner, Ulrike, Niels Brüggen, Peter Gerlicher, Mareike Schemmerling, und Christa Gebel. 2013. «Zusammenfassung der Teilstudie: Identitätsarbeit und sozialraumbezogenes Medienhandeln in Sozialen Netzwerkdienste. Im Rahmen der Untersuchung «Das Internet als Rezeptions- und Präsentationsplattform für Jugendliche»». 2013. https://www.jff.de/fileadmin/user_upload/jff/projekte/konvergenzstudien/JFF_Kurzfassung_Teilstudie_Identitaetsarbeit.pdf.

Wilkens, Andreas. 2009a. «Facebook gründet Sicherheitsbeirat». heise online 07.12.2009. https://www.heise.de/newsticker/meldung/Facebook-gruendet-Sicherheitsbeirat-878471. html.

Wilkens, Andreas. 2009b. «Facebook verändert Kontrolle über Privatsphäre». heise online 10.12.2009. https://www.heise.de/newsticker/meldung/Facebook-veraendert-Kontrolleueber-Privatsphaere-881817.html

Wittenberg, Jochen, Markus Poguntke-Rauer, und Martin Ragg. 2001. «Vorsichtig optimistisch. Chancen und Risiken des Internet aus Sicht der Experten - Ergebnisse einer Online-Befragung». In Sozial@rbeit online, herausgegeben von Oliver Poseck, 39-69. München: Luchterhand.

Witzel, Andreas. 2000. «Das problemzentrierte Interview». 2000. http://nbn-resolving.de/ urn:nbn:de:0114-fqs0001228. 